\title{
LA VILLA A MOSAÏQUES DE MIENNE-MARBOUÉ (Eure-et-Loir)
}

\author{
par Michèle BLANCHARD-LEMÉE
}

La villa à mosaïques de Mienne--Marboué a connu une certaine célébrité au xix ${ }^{e}$ siècle, peu après sa découverte. Puis, an même temps que les vestiges dégagés par la fouille s'effaçaient, un oubli relatif est tombé sur le site. Oubli mérité si l'on s'en tient aux conclusions historiques développées jadis. Oubli provisoire, je l'espère, si l'on accepte les conclusions de la présente étude ${ }^{1}$.

La commune de Marboué se situe à $4 \mathrm{~km}$ au nord de Châteaudun; le bourg est massé le long de la R.X. 10 et surtout à l'est de cette route. Le hameau de Mienne est à l'ouest de la route, au bord du Loir, bien abrité des vents du nord par un coteau boisé; en 1834, Mienne ne comptait que quatre maisons. On procédait alors au redressement du tracé de la route d'Espagne et à la construction d'un nouveau pont sur le Loir. Les habitants du hameau vendirent à l'entrepreneur les pierres et les tuiles qu'ils trouvaient en abondance dans un terrain inculte leur appartenant en commun : ils en écoulèrent 50 tombereaux, avant de tomber, en mai 1834, sur un pavement trop dur pour être dépecé. C'était la mosaïque à inscription. Alerté à la fin de juin, l'ingénieur des Ponts-et-Chaussées et amateur d'archéologie Louis-Gustave Guérineau de Boisvillette acheta la mosaïque; puis il obtint du Conseil Général l'autorisation et les moyens de mener des fouilles sur presque tout le site de la villa et de ses dépendances². Ces fouilles eurent lieu en 1834 et 1835) et donnèrent lieu immédiatement à des publications partielles par Guérineau de Boisvillette lui-même ${ }^{3}$ et par un érudit orléanais, C.-F. Vergnaud-Romagnesi. Le Mémoire de ce dernier est

1 Les principaux aspects de co dossior ont ete présentes à la Sociéte nationale des Antiquaires de France, le 2 mai 1979. Je liens a exprimer ma reconnaissance à tous les savants qui ont bien voulu mencourager et me conseiller : II me X. Gauthier at .II. IR.-II. Bautier, Dom J. Dubois, N. Duval, I. IPleuriot, I.. Pressouyre, le R. P. Van der Straten ; que soirnt aussi remercies ici lous coux qui mont facilite lacces aux documents: Mme Rolland al Mu. (i. Cabon, I. Ighmouracine, 1). Joly, B. Iegrand et I. Lizier.

2 Avec l'autorisation du propriétaire de la plupart des terrains ; seule cechappa a la fouille une parcelle recollvant l'aile nord de la villa (cf. fig. 1 !.

3 Nolice sur les monuments el la mosaïque trouc'és à Mienne, près Marboué (Eure-el-Loir), dans Mémoires de la Société royale des Anliquaires de France, XII, 1836, p. 153-173, pl. IV cité ci-dessous Nolice.

4 C.-H. Vergnat d-Romagxesi, Mémoire sur une mosaïque el des anliquilés romaines lrouvées prés de Châteaudun (Eure-el-Loir), dans Annales de la Société des Sciences, Belles-Lellres el Arts d'Orléans, XIII, 1835, p. 192-211, et Addilion au Mémoire..., ibid., p. 293-296. Résumé par l'auteur dans Bullelin Monumental, 1, 1834, p. 271-274 jeité ci-dessous Mémoirè, 


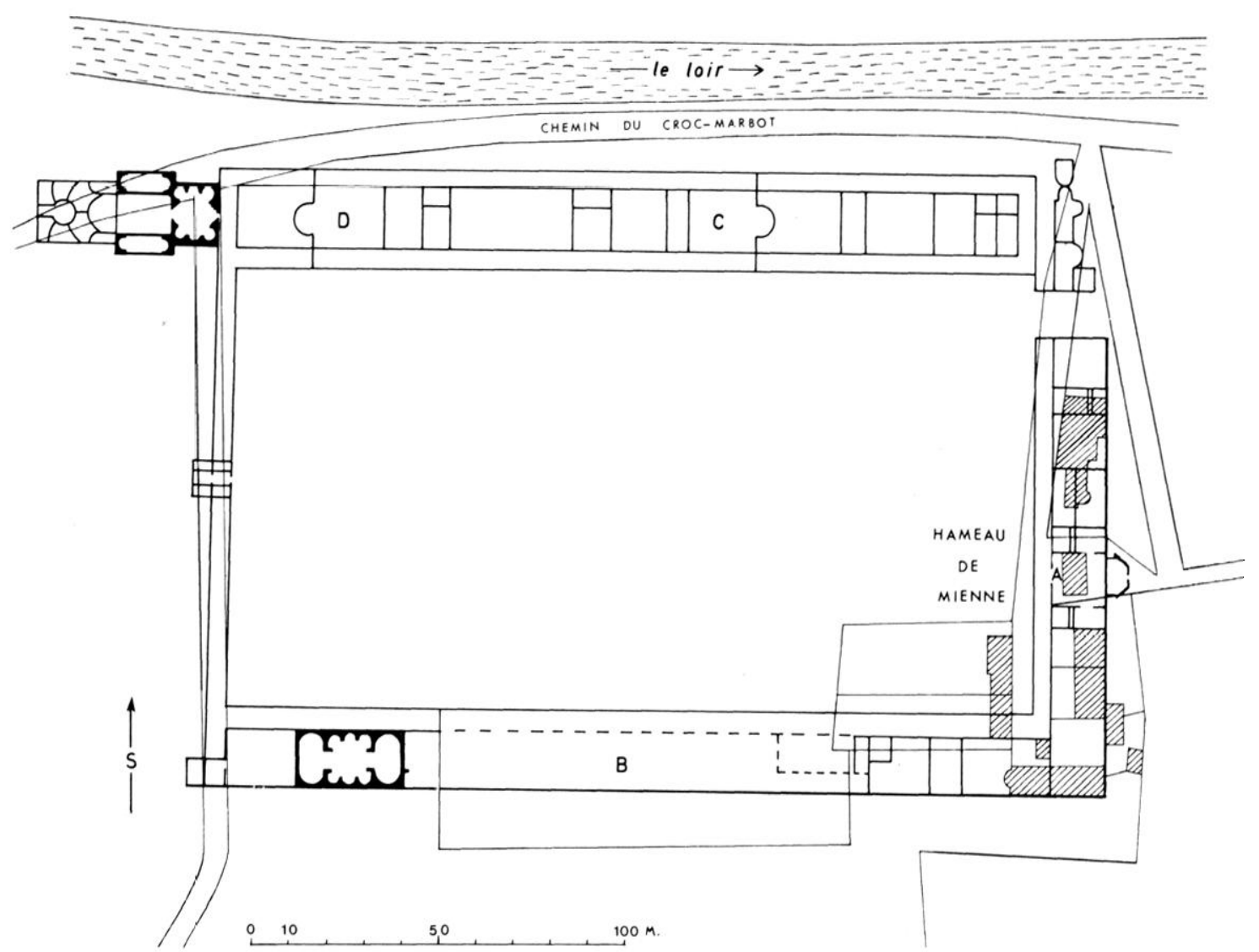

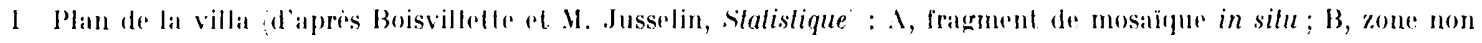

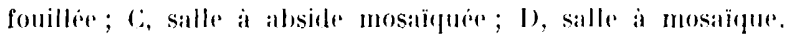

illustré de plans provisoires des édifices et des dessins de deux mosäques, tirés de relevés communiqués par Boisvillette, qui ne joignit lui-mème à son article qu'une lithographie du calque du panneau à inscription de la première mosä̈que. Trente années après, le fouilleur publiait une brève synthèse dans son ouvrage Slalislique archéologique d'Eure-el-Loir, avec un plan définitif mais très réduit de la villa ${ }^{5}$.

Un troisième érudit, $H$. Lejeune, conservateur de la Bibliothèque de Chartres (1771-1858), membre fondateur de la Société des Antiquaires de France, avait constitué sur la villa un dossier nourri de nombreux croquis qui a péri dans l'incendie de la Bibliothèque municipale de (hartres en 1944. La substance en est heureusement transmise et enrichie par un autre manuscrit, celui de I'Invenlaire archéologique dEure-el-Loir, de l'archiviste départemental Maurice Jusselin ${ }^{6}$.

Les fouilles de Boisvillette ont fait apparaitre les fondations d'une vaste résidence rectangulaire $(150 \mathrm{~m} \times 240 \mathrm{~m}$ ) (fig. 1), dont les bâtiments encadrent sur trois côtés une cour

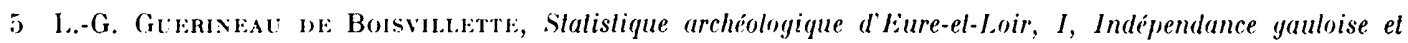
Gaule romaine, Chartres, 1864 , p. 267-273; plan de la villa p. $26 x$ cile ci-dessous slatistique:.

6 Cinq cahiers conservés à la Direction des Archives departementales d'Eure-et-Loir ; pour Marboue, cf. Cahier no III. Trxle reproduit en partie dans H. I.l\%kr, Varbour, cile hislorique, site touristique, llarboué, 1979, p. 93-132. 
à péristyle. F. Grenier ${ }^{7}$ classe le plan de la villa urbana de Mienne dans le type "à cour antérieure». La galerie orientale n'est en effet précédée d'aucune salle et l'entrée se faisait par un portail monumental; le bâtiment principal se situait face à l'entrée, la grande salle pavée de la mosaïque à inscription à peu près dans l axe du portail. Nais les pièces d'apparat à absides et mosaïques (salles $C$ et I)) se répartissent sur toute l'aile méridionale, elle-même prolongée par des thermes; la partie de l'aile nord ${ }^{8}$ symétrique à la salle I) était occupée, semble-t-il, par des installations thermales; on trouve encore d'autres thermes, plus petits, à l'angle sud-est de la villa. Toute la colonnade paraît donc avoir èté entourée de pièces à usages sensiblement équivalents. On se rapproche du plan "à péristyle", ainsi que le suggèrent d'ailleurs les comparaisons avancées par F. Grenier avec les villas de Bignor et Woodchester en Grande-Bretagne; or, I. A. Richmond considère ces deux villas comme des exemples de véritable "courtyard", cour intéricure complète ou incomplète9. R. Agache a insisté récemment sur la rareté de ce plan dans les grandes plaines du Yord de la France. Seul le palais de Vieux-Rouen-sur-Bresles peut se comparer, par son plan, ses dimensions $(65 \times 95 \mathrm{~m})$ et par l'association d'une galerie de façade à la cour à péristyle, à la villa de Mienne ${ }^{10}$. Des villas "à péristyle" ont été détectées par des prospections aériennes en Bourgogne et en Berry"

Bien que le plan de la villa, tel qu'il apparait sur le relevé schématique de Boisvillette, ait une apparence cohérente. il convient de se demander si les diverses dispositions qu'il réunit ne résultent pas d'aménagements successifs, voire séparés par plusieurs siècles. Les éléments de datation archéologique nous font défaut, car tout vestige a disparu sur le terrain; du petit matériel, très rare, recueilli lors de la fouille, presque rien ne subsiste ou n'est identifiable au Musée de Châteauduñ ${ }^{12}$.

\section{Dalalion.}

Pour les contemporains de la découverte, c'était la splendeur romaine des premiers siècles de l'Empire qui ressuscitait sous leurs yeux, dans cette partie de la cité des Carnutes dont le passé restait alors totalement obscur jusqu à la fin du ve siècle. Vergnaud-Romagnesi attribuait l'établissement aux règnes de Tibère, Claude ou Néron et sa destruction à

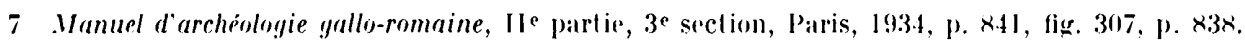

8 In lotissement moderne occupe la parcelle non fouillé par Boisvillette ; la Societe Inunoise a effectue des prospections lors des travaux : ne subsistaient plus que les fondations des murs exterieurs; le seul materiel recuedli consista ph deluris de tuiles.

9 Dans A. I. F. Raver, The Roman Villa in Brilain, Londres, 1969; Norlh I.righ, fig. 2.4, p. 61, 13ignor,

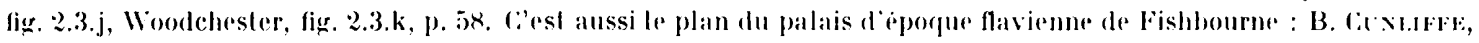
Fishbourne, 1 Roman Palace and ils Garden, 1971, fig. 13, p. 75).

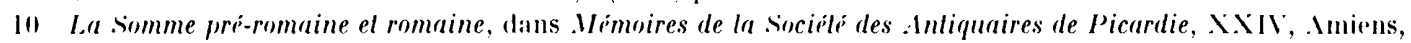
$197 x$, p. 296-298, plan p. 297. Lauteur rapproche celte villa de celles de Sainte-Marguerite Seine-Maritime el de saint-Lirich Moselle.

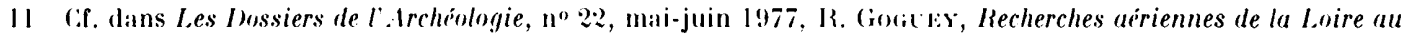

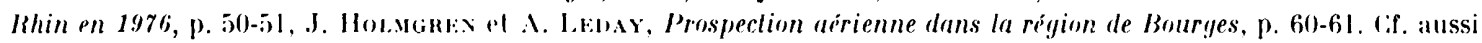
J.-G. Goncis, Les villas hispano-pomaines, p. 113-119, en particulier p. 117-11

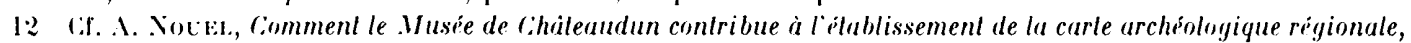

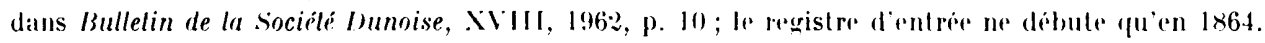




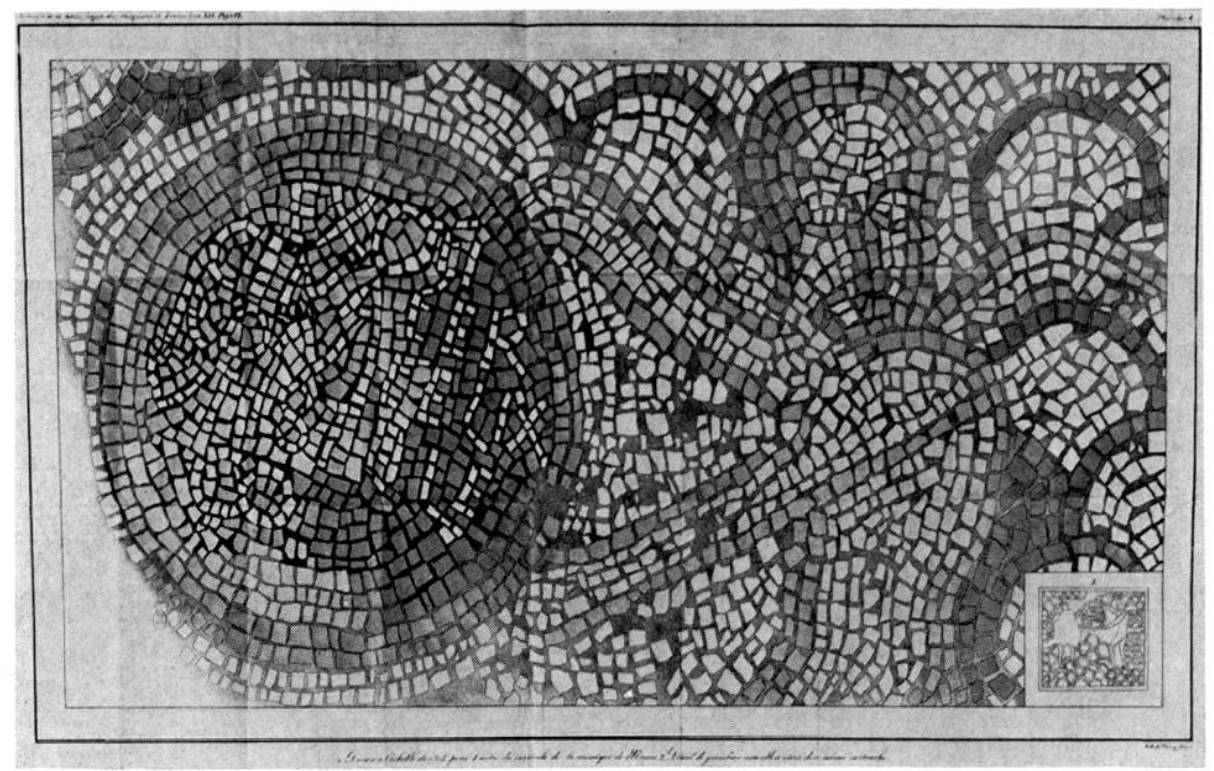

2 L'inscription sur mosaique d'apres Boisvillette, Notice; et l'un des génies.

l'invasion de 40713. Boisvillette proposait, pour la construction, une fourchette entre te $\mathrm{I}^{\mathrm{er}}$ siècle et la fin du II $^{\mathrm{e}}$ siècle. avec une préférence pour les deux premiers siècles et une durée de survie jusqu'aux $\mathrm{x}^{\mathrm{e}}$ et $\mathrm{x}^{\mathrm{e}}$ siècles ${ }^{14}$; mais il n'envisageait pas qu'aucun élément de la villa pût être attribué à une période postérieure à sa fondation. Or il est possible de démontrer qu'une partie du décor peut faire l'objet d'une datation beaucoup plus tardive qu'on ne l'avait précédemment pensé.

\section{L'inscriplion sur mosaïque.}

L'élément de datation le plus important reste l'inscription sur mosaïque provenant de la grande salle centrale de l'aile occidentale. Nous en avons la reproduction aux dimensions réelles par la lithographie en couleurs publiée par Boisvillette ${ }^{15}$ (fig. 2) et reproduite en noir et blane par Vergnaud-Romagnesi (fig. 3). L'inscription est contenue dans un médaillon de $27, \bar{i}$ à $28 \mathrm{~cm}$ de diamètre présenté par deux génies ailés. Le fond en est polychrome : "un quart du cartouche est rouge, un autre quart noir, et, dans le centre, les deux couleurs se mêlent par des teintes graduées ", décrit fort bien Vérimée ${ }^{16}$.

Les lectures et a forliori les interprétations de l’inscription ont éte aussi nombreuses que variées.

13 Mémoire, p. 203 el 206.

14 Notice, p. 169-170 et 172.

15 Notice, pl. IV hors-texte et p. 158.

16 Notes d'un voyage dans l'ouest de la France, Paris, 1836, p. 38 (rapport au Ministre de l'Intérieur, où P. Mérimée préconise la dépose de la mosaïque: 
Texte de l'inscription de Steleco ?d'apres VerguandRomagnesi, Mémoiré.

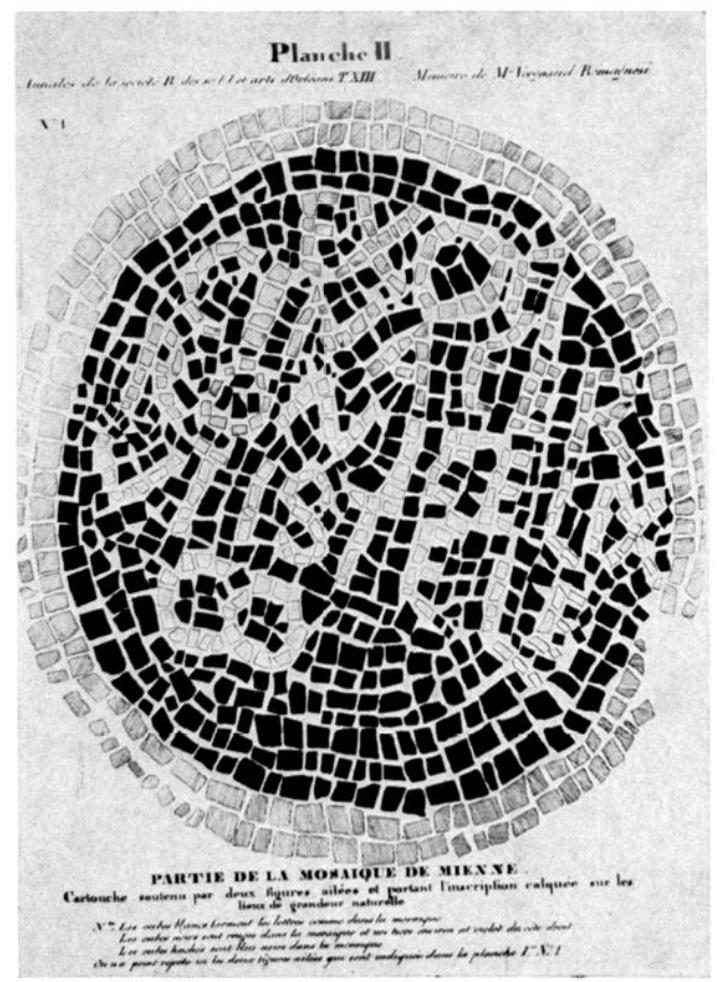

La lecture la plus communément acceptée, proposée par Boisvillette ${ }^{17}$. reprise par le C.I.L.18 et par A. Blanchet dans I'Inventaire des mosaïques de la Gaule ${ }^{19}$ est la suivante : EX OFI/CIXI FER/ RONI FELIX/U'T ISTE LE/GO, ex officina Ferroni, felix ut isle lego; ce que Boisvilletle traduit ainsi : "De l'atelier de Ferronius, aussi heureux que lui, je te lègue », ou mieux : "Eleve de Ferronius, je lègue à la postérité cet ouvragre digne de lui ». On constatera que la traduction est des plus difficiles et nécessite une interprétation très large. D'autre part l'avant-dernière lettre est certainement un $C$, non un $G$.

A. de Caumont ${ }^{20}$ proposait de lire felix ut isle leco, renonģant à toute traduction. Pour sortir de cette impasse, F. Lejeune faisait de chaque lettre ou groupe de lettres l'abréviation d'un longr mol. pour étayer sa thèse de l'édification de la villa par le Consul Memmius, après une victoire sur les barbares en $343^{21}$. P. Mérimée préférait, quant à lui, une interprétation plus sentimentale ${ }^{22}:$ ex officina Ferroni(i), Felix, Clistel(a)e co(njugi), traduisant : "ouvrage de Ferronius : Felix a Clistela sa femme».

Mais il ne manquait pas de mentionner ${ }^{23}$ l'opinion de Ch. I.enormant, qui lisait la fin felix uli. Sleleco, soit "Ouvrage de Ferronius ; a Stelechus, qu'il s'en serve heureusement » et voyail dans le dernier mot le datif d'un nom formé en chus, c'est-à-dire d'un nom grec romanisé.

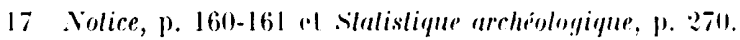

ix r.I.L. X, $\mathrm{n}^{\circ} 30162$.

19 Paris, 1909, n" 932 "l planche.

20) Notice sur les villae ou maisons de campagne gallo-romaines, dans Bullelin .Monumental, VI, 1×.11, p. IX6.

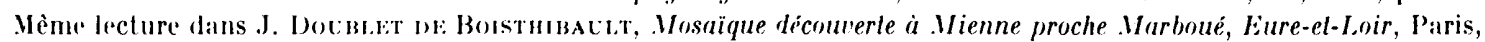
1836, p. 77-79, reproduit par (: L.1:Flake, Annuaire d'Eure-el-Loir pour 184:, p. 23-25.

21 Mémoire presenti an avril $1 \times 40$ à l'Academie des Inscriptions el Belles-leettres, refuse al reste inedit; cite par M. Jusselin, f" 76.

220 op. cil., p. 3x.

23 Itid., p. 39. 
J'adopterai pour ma part une lecture très proche de celle de Ch. Lenormant: ex officina Ferroni (signature du mosaïste), felix uli (déformation de la formule propitiatoire Ulere felix par substitution de l'infinitif à l'impératif),

Steleco nom réel du personnage auquel s’adresse ce vœu, au vocatif comme il se doil après une acclamation.

La formule ex officina semble s'être introduite assez tardivement en mosaïque : ainsi aucun des exemples recensés à ce jour, au nombre de six, quatre en Afrique ${ }^{24}$, deux en Espagne $^{25}$ ne peut remonter plus haut que le rve siècle. A Khenchela et à Tossa de Mar, la signature de l'officine s'accompagne comme à Vienne d'une formule propitiatoire donnant, dans le second cas, le nom du propriétaire. Le nom - très rare du mosaïste Feronius ou Ferronius était à l'origine, selon Schulye un gentilice italien dérivant du nom de la réesse Feronia. L'acclamation utere felix, très répandue sur les petits objets usuels, est moins f́réquente sur les éléments d'architecture ${ }^{27}$. Je n'en connais en mosä̈que que deux exemples. une fontaine de Carthage ${ }^{28}$ et un pavement de Teano où l'inscription est christianisée $e^{29}$.

Le nom du propriétaire Steleco n'est ni romain, ni celtique ${ }^{30}$. On ne peut manquer de le rapprocher du nom du Maitre des deux Milices Stilicon, dont le père était vandale. Ceest en effet un nom germanique, variante de l'hypocoristique Stilke formé sur le radical Stilli (calme, tranquille) avec le suffixe $K^{31}$. C'est un nom rare, dont le seul exemple relevé jusqu'à présent en Gaule est apporté par une Charte de Cluny datée de $814^{32}$. Les lectures antérieures de l'inscription sur mosiäque araient done dissimulé le fait essentiel que le propriétaire de la villa de Mienne-.Iarboué, au moment où fut posée la mosaïque, portait un nom germanique. Ceci laisse ouvert un large éventail de possibilités de datations à partir de la fin du ive siècle.

24 C. S. Germax, Les mosäques de Timgad, Paris, 1969, $\mathrm{n}^{\mathrm{n}} 231$, arec bibliographir, ret J. Lassc's, Recueil de Nolices el Mémoires de la Sociélé archéologique de la Wilaya de Constantine, J.XXI, 1969-1971, p. 49-50, fig. 9.

25 Mosaïque dionysiaque d'Annius Ponnius à Merida, maintenant attribure au plus tard au début du ve siecle en raison de trouvailles monétaires, A. Bi.a.co Fressizo, Mosaicos romanos de Merida, Madrid, 1978, p. 21,34,pl.26. Mosaïque de la villa de Tossa de Mar Catalogne altribuer aux ve ou ve siecle, A. BAt.r., dans La mosaïque grécoomaine, I, Paris, 1965, p. 33, 36-37, fig. 13.

26 11. Scuct.s, Zur Geschichte der Iateinischen Eigennamen, Göltingen, 1904, p. 165.

27 ․ Juval., Plastique chrótienne de Tunisie el d'Algérie, dans Bullelin Archóologique du Comilé des Travaux Misloriques abrége : B.C.T.H. , n ${ }^{11 e}$ série, $\times, 1972$, p. 116 , fig. 66 b, sur un pilier de Khenchela ; signalons un usage plus paradoxal de la formule, sur un sarcophage de Tipasa 'M. Cunistofle, Rapporl sur les trabaux de fouilles el de consolidations..., Alger 1938, p. 79.

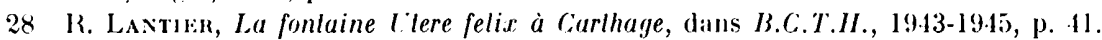

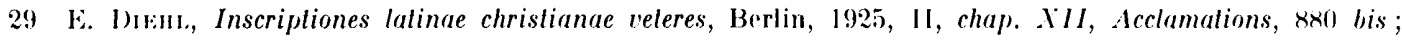
la christianisation de la formule a assure sa survivance : on ne peut donc l'ériger en ilement de datation.

30 I. Fleuriot a bien voulu me confirmer l'absence de loute racine celtique à ce nom el son rattachement all mot pan-germanique slilli.

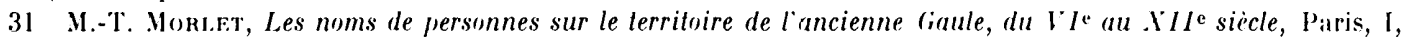
1968 , p. $x$ et p. 203. E. Forstriax, dildeutsches Namenbuch, J, Jersonnennamen, Bonn, 1900, s.v. Stilico.

32 Sous la forme stilligo : cf. II.-T. Mortet, op. cit., p. 203. I.es recueils de F. Fonstemaxi, op. cit., et

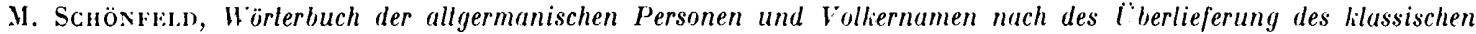
Alterlums bearbeitel, Heidelberg, 2 e ed., 1965, p. 209-211, ne citent que le Maìre des Milices Stilicon. l.e nom est absent du fichier onomastique de l'Institut historique allemand de Paris. 
La forme des lettres de l'inscription est essentiellement tributaire de la technique très médiocre de la mosiäque ${ }^{33}$. Cependant l'accumulation de certaines caractéristiques - L obtus dont la barre passe sous la voyelle suivante. N penché proche du V très ouvert, opposition entre des lettres larges comme le 0 et le ci tres ronds et des lettres etroites comme le E, le F. le T nous renvoie à des exemples de la fin du ve siècle ${ }^{34}$. Le F a trois barres ligne 1) semble avoir été en faveur en Gaule aux rve et ve siècles ${ }^{35}$.

\section{La mosaïque de Slelec'n.}

L'analyse stylistique du parement apporte des arguments décisifs en faveur d'une datation tardive ${ }^{36}$ esquissér par l'étude paléographique. Nous disposons d'un certain nombre de documents graphiques. Je relevé schématique (fig. 4) publié par VergnaudRomagnesi ${ }^{37}$ doit ètre complété mentalement pour rétablir l'ordonnance d'ensemble, à l'aide du plan de l'aile occidentale (fig. 11). Ces planches ont été gravées d'après les relevés exécutés pour Boisvillette par le conducteur des travaux Fillon ${ }^{38}$; M. Jusselin avait photographié un détail du dessin original de Fillon qui differe un peu de la gravure ${ }^{39}$.

Le pavement lui-mème a disparu dans les années qui ont suivi sa découverte. Lin 18:36 la commune de Varboué avait cédé gratuitement le terrain au Conseil Cénéral d'Eure-etLoir, qui arait fait édifier un hangar sur la mosaïque. Vais, dès 1834. un habitant seétait fait réserver le droit de faire visiter la mosaïque; nous savons par un article indigné d'A. de Caumont qu'il en ravivait les couleurs par de fréquentes aspersions, qui nont pas tardé à déchausser les tesselles. 1)es 1842, la perte était consommée ${ }^{40}$. In fragment de l’angle sud-est $(73 \times 99 \mathrm{~cm})$ de la pièce arait cependant échappé à la destruction : retrouvé lors de la construction d'une maison en 1908. il fut soigneusement isole et préservé par un puits maçonné profond de $89 \mathrm{~cm}$ et fermé d'une dalle de pierrete

Le pavement mesurail environ $11.10 \mathrm{~m}$ sur $13,30 \mathrm{~m}$. Le canevas était centré et le centre de la pièce marqué par une larune. ce qui a fait supposer à Boisvillette l'existence d'un cippe à cet endroit.

Le compartiment central de la mosilïque $(0.68 \mathrm{~m} \times 0.80 \mathrm{~m})$ est, chargé d'un molif orthogonal et

33 Les tesselles hlanches des lellres, irregulieres ef mal jointes, mesurent de 4 a $11 \mathrm{~mm}$ de coilt; celles du fond peurent atteindre 12 a $15 \mathrm{~mm}$ sur leur cotr le plus long; Jes lignes ondulent jusqu it se chevalucher.

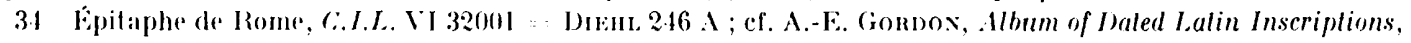
11I, Rome and the Neighbourhool, 1.I. 200-5:5, Berkeley, 1965, pl. 176 a, p. 177-17x.

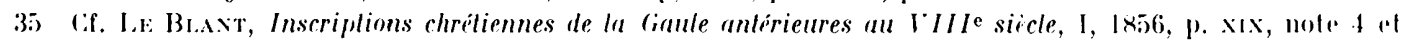
p. xxiv, note l Rome, inscr. de 139 on li2.

36 A. Bravenet, La mosaique, Paris, 1920, p. 66, écrivait : "si l'on considere le style réellement barbare déjà de cette mosiägue de Nienne, on ne verra aucun olstacle it la faire descendre alu moins jusqu'i la fin du we siecle"

37 Op. cit., pl. I, 1 ; plan de l'aile occidentale pl. I, :2.

38 Renseignement donne par .I. Jusselin, cahier III, fo 91 ; le releve original de Fillon devrail se trouver

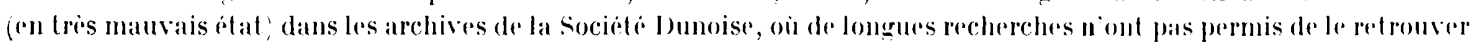
entré le 24 II I $1 \times 65$ an Vuséde de la Socielí, n" $491 \mathrm{R}$; échelle $1 / 10,1,31 \mathrm{~m} \times 1,13 \mathrm{~m}$.

39 Ibid., fo 92 ; dimensions du cliché : $12,3 \mathrm{~cm} \times 16,8 \mathrm{~cm}$. Notre fiğure est un calỵue effech ure par R. Prudhomme.

40) Ms. Jusselin, fo 93, 107-109: Boisvilletle avail achete la mosaïfue 3 F lal loise soil entiron 2 m $^{2}$ aux

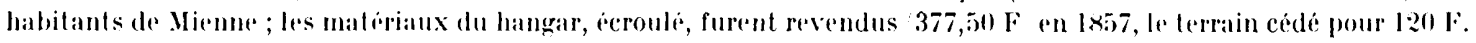

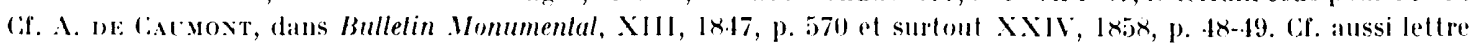
de Fillon à Boisvillette, du 19.1.1862, Archives de la société Archéologique d'Eure-el-Loir, no 1224. La mosaingue a toutefois ale inscrite sur la premiere liste de .10muments Historigues élatorée en $1 \times 53$.

41 A. Braxchet, Int. Mos. I, n"932, signale dubitativement la redecouverte du fragment ; Vs. Jusselin, fo 107. 


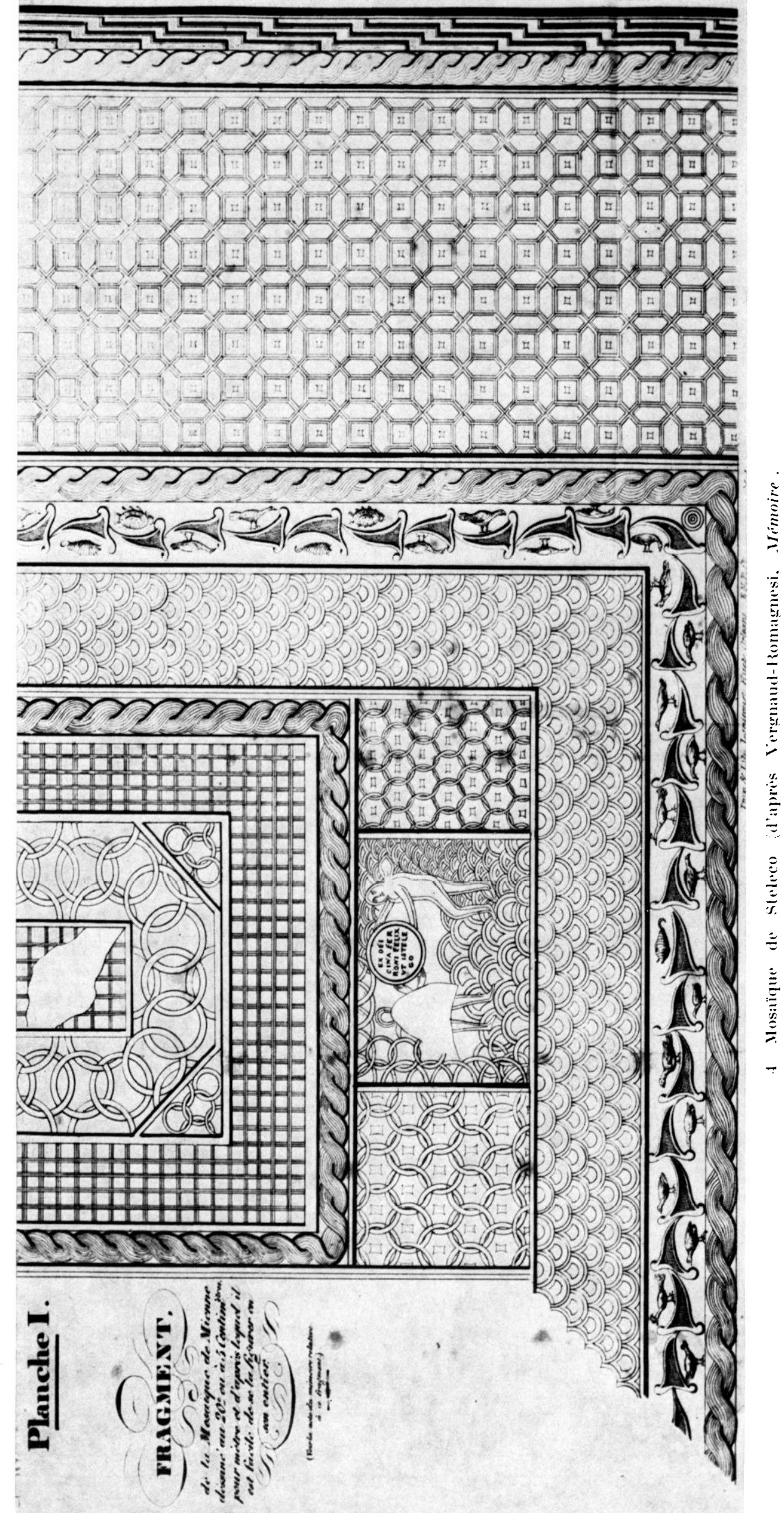


uniforme : un quadrillage de filets doubles à cases petites (environ $8 \mathrm{~cm})^{42}$. Le rectangle central est inclus dans un hexagone lui-mème inscrit dans un carré de $1,92 \mathrm{~m}$ de còté, et orné d'une composition orthogonale de cercles sécants; je ne saurais dire si l'irrégularilé des intersections des cercles est due à la réalité ou à la maladresse du graveur.

Le carré chargé d'un hexagone est emboité dans un carré de 3,35) m environ de còté bordé d'une tresse à deux brins $(20 \mathrm{~cm})$; la partie visible, une bande de $0,55 \mathrm{~m}$, est couverte du mème quadrillage que le compartiment central. Ce troisième carré est flanqué de deux rallonges rectangulaires (environ 1,15) $\mathrm{m} \times 1,92 \mathrm{~m}$ ) qui adaptent le schéma centré à la forme rectangulaire de la pièce. Du còté de l'abside, on restitue un seul panneau a motif uniforme.

Face a la porte, la rallonge se subdivise en trois panneaux inégaux ; les deux rectangles latéraux dissvmétriques sont couverts de cercles sécants déterminant des six-feuilles; le panneau médian $(0,80 \mathrm{~m} \times 1 \mathrm{~m})$ est celui de l'inscription. L'ensemble constilué par le carré central el les deux rallonges s'emboîte dans deux autres rectangles emboîtés, qui forment une série de bandes concentriques au pourtour de la pièce. La première bande $(0,60) \mathrm{m}$ a $0,70 \mathrm{~m})$ est ornée d'écailles au trail chargées d'écailles de couleur emboitées. Elle est séparée de la suivante par une double bordure composée successivement d'un rinceau et d'une tresse à deux brins.

Le rinceau (large d'environ (0.35) m) est constitue de gaines d'acanthe en forme de cornes superposées selon les rourbures d'une sinusoïde. Les concarités sont meublées d'animaux, "diseaux. tortues, poissons, scorpions", selon Vergnaud-Romagnesi43. "des oiseaux. des poissons", selon Mérimée $e^{44}$. Les volatiles sont de loin les plus nombreux, les scorpions et les tortues sont sans doute des animaux marins mal compris par Veronaud-Romagnesi et par Fillon.

La bande périphérique $(2,0: 5 \mathrm{~m}$ a $2,10 \mathrm{~m}$ de largeur était uniformément couverte d'un motif d'octogones sécants et adjacents. Le fragrment conservé in silu montre que le dessinateur a régularisé les octogones et qu'il s'ag̣it en fait d'octogones irréguliers sécants et adjarents par les longrs còtés, qui déterminent des hexagones oblongs assez trapus $(10 \mathrm{~cm} \times 20,5 \mathrm{~cm})$ et des carrés exigus (fig. 5). Le canevas est tracé par des filets doubles noirs sur fond blanc ; des motifs colorés s'emboîtent dans chacun des motifs au trait, en rouge dans les carrés, en rouge ou en gris dans les hexagones oblongs.

La bordure d'ensemble du pavement est double. On trouve d'abord une bande noire large de $16 \mathrm{~cm}$ comportant un brin rouge et un brin gris (fig. 5); vient ensuite un méandre fractionné a fractions rouges et grises. Enfin Vergnaud-Romagnesi parle d'une "large bande rouge " 45 entourant le parement: : c'est, la bande de rarcord, que le dessin traduit par une étroite bande noire.

La structure du pavement, compartimenté en de multiples panneaux, parfois juxtaposés, le plus souvent emboités, témoigne d'une conception de l'espace assez éloignée de l'organisation classique des mosaïques romaines. La mosaïque de Vienne combine d'une part la juxtaposition de surfaces à motifs disparates séparés par de simples filets, type de composition qui apparaît au ve siècle, notamment dans la villa de Loupiac --- aux angles des galeries bordant la piscine $\quad \mathbf{4 6}^{46}$ et dans l'église Sainte-Marie-de-Terrassa en Catalogne ${ }^{47}$, avec d'autre part une construction en emboîtements de tapis, construction connue, non loin de Marhoué, par l'une des mosaïques de Souzy-la-Briche postérieure au début du

42 Le dessinateur rend par des traits fins les joints entre les rangés de tesselles dans les contours des motifs : comparer le dessin a la photographie du fragment in silu.

43 op. cit., p. 199 .

4.4 Op. cil., ए. 37.

45 Op. cil., p. 199.

16 Cadillac, aspects connus el inconnus diun canlon, par la Commission régionale d'Inventaire d' Iyuilaine, Bordeaux, 1977, p. 9 , relevé de C. Ney, service d'Architecture antique.

47 X. Barrat, les mosä̈ques de l'ancien siége épiscopal d'Egara (Terrassa) en Calalogne, dans La mosaïque yréco-romaine $I I$, Paris, 1975, p. 25l-254, pl. Xi:. 


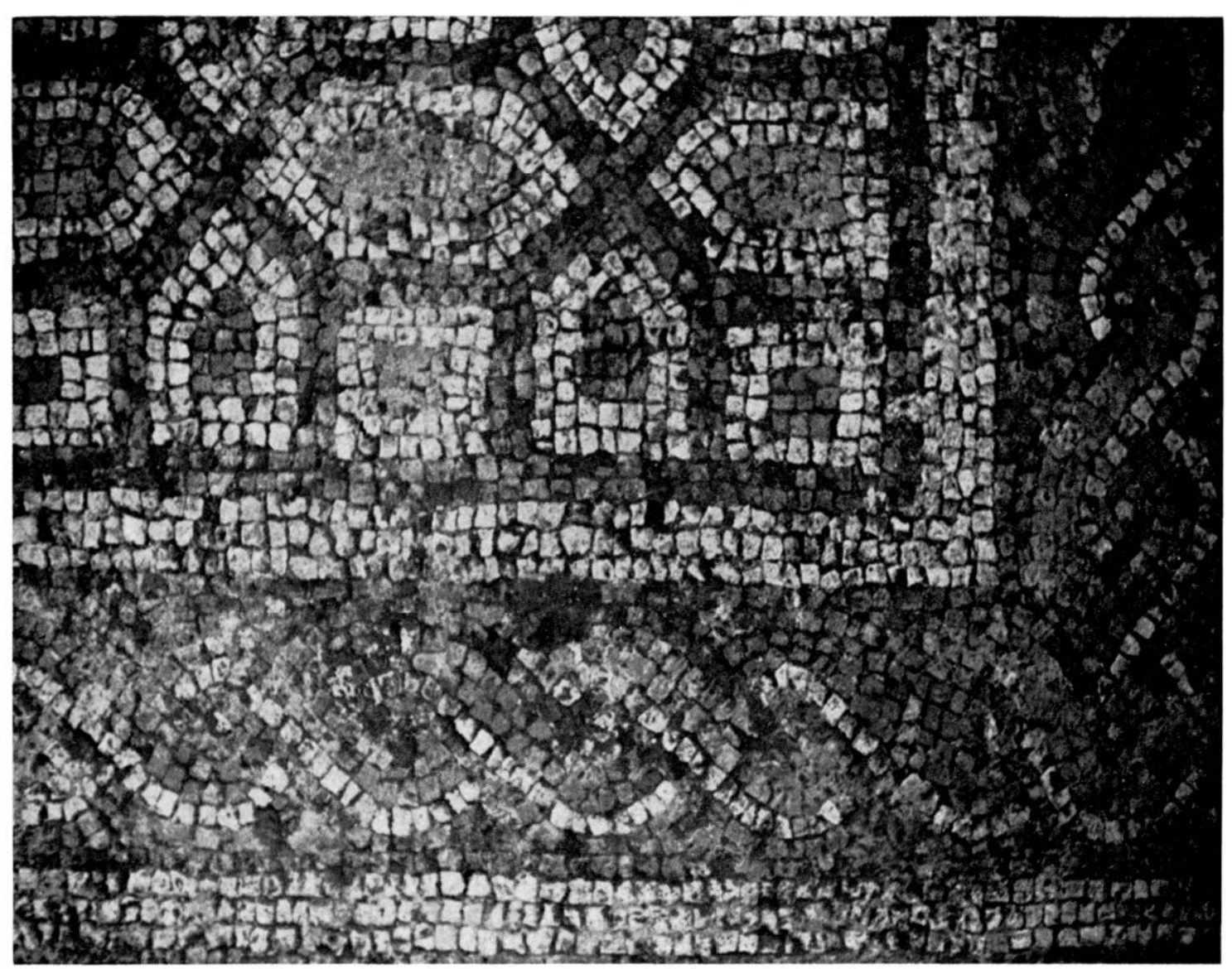

5. Fiagment in silu.

Iv $^{\mathrm{e}}$ siècle ${ }^{48}$. Nous pouvons nous faire une idée de la farture du pavement par le calque de l'inscription publié par Boisvillette (fig. 2), par le fragment in silu (fig. 5) et par de brèves notations des auteurs du xixe siecle.

L'inscription, dans laquelle la densilé des tesselles ne dépasse pas 10: tesselles par dm². reste cependant la partie la plus soignée de la mosäque; dans les personnages, la densité lombe a 75) tesselles par $\mathrm{dm}^{2}$, dans le fragment de décor géométrique conservé elle varie entre jo et bo par $d n^{2}$. La palette est restreinte : un noir grisàtre. un blanc creme. un gris beige, un rouge, dans des calcaires tris ternes. Vergnaud-Romagnesi ne signale que quatre couleurs pour tout le pavement. blanc, jaune, bleu noir et rouge ${ }^{49}$ : Mérimée mentionne l'existence d'un rose ${ }^{50}$, dont la présence est attestée par la lithographie publice par Boisvillette. Le support a été décrit par Boisvillette : les cubes "sont liés par un mortier blanc tris fin formant une courhe de 0,(12) m a 0,(125) m plarée sur une aire de ciment épaisse de $0,08 \mathrm{~m}^{51}$.

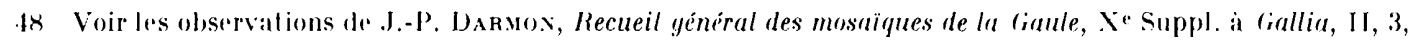

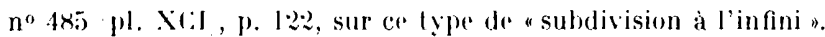

49 Op. cit., p. 199 ; ses identificalions de matériaux sont fantiisistes : les tesselles blanches et noires sont en calcaires 'd'origrine non identifiable.

50) Op. cil., p. 37 .

51 Notice, p. 155: description confirmé par un fragment de quatre lesselles conserví par la sociéte Inunoise: lit de pose des tesselles en chaux pure, sur une couche de morlier de luileau tries fin. 
C'est par les mêmes documents que nous pouvons approfondir l'analyse du style ornemental et figuré de la mosaïque. Le traitement du canevas d'octogones sécants et adjacents, avec un motif emboité et uniformément coloré dans chaque motif, constitue un indice de datation tardive. Le même canevas, traité de manière identique, se retrouve en Tarraconaise, dans le pavement de l'église Sainte-Iarie-de-Terrassa que X. Barral attribue, après P. de Palol, à la seconde moitié du ve siècle ${ }^{52}$; d'une manière générale, ce procédé, donnant un effet de "coloriage " des motifs, est fréquent dans les mosaïques de la Basse-Antiquité du Sud-Ouest, notamment à Taron ${ }^{53}$ a a Ilontraret et à Loupiac ${ }^{54}$; en Tarraconnaise, la villa de Tossa de IIar en apporte un autre exemple ${ }^{55}$. Dans d'autres écoles de mosaïstes florissantes dans l'Antiquité tardive, par exemple à Ravenne. le procédé stylistique est quelque peu différent : les motifs emboités sont cernés d'un filet simple noir ${ }^{56}$.

Le rinceau en gaines d'acanthe superposées dépourvu de toute allusion au végétal n'a pas d'équivalent en mosaïque; on peut toutefois le rapprocher des paires de rinceaux croisés en gaines d'acanthe qui eneadrent le personnage central de la mosaïque à inscription de Tossa de Mar ${ }^{57}$.

Les deux génies nus et ailés qui portent le cartouche de linscription s'enlèvent sur un fond d'écailles imbriquées au trait. La photographie du relevé de Fillon (fig. 6) permet de rectifier les inexactitudes de lillustration de Vergnaud-Romagnesi. Le graveur a interprété comme la juxtaposition de deux motifs (ce qui serait un caractère stylistique médiéval) une simple maladresse du mosaïste dans le tracé et l'orientation du motif d'écailles à gauche du tableau. Quant aux deux génies, ce sont de simples silhouettes, aux contours raides et aux traits à peine esquissés; le modelé, esquissé sur l'épaule par le jeu des tesselles roses et jaunes, est nul ailleurs (fig. 2). Il est difficile de tirer des conséquences chronologiques du caractère naif de ces figures; des traits "primitivistes" ou "populaires " apparaissent dans les représentations humaines de mosaïques provinciales datées du ive siècle ou du début du ve siècle, notamment en Bretagne le cas le plus extrême étant peut-être celui de la Vénus de Rudston ${ }^{58}$. et en Espagne, avec la mosaïque d'Annius Ponnius de Merida ${ }^{59}$.

Le thème de l'imago clipeala ou de l'inscription dans un clipeus porté par deux génies est passé, vers le milieu du ive siècle semble-t-il, de l'art triomphal ou funéraire au décor des objets luxueux appartenant à l'aristocratie romaine, tels le coffret de Projecta ${ }^{60}$ ou le

52 Les mosaïques romaines el médiévales de la regio laielana, Barcelone, 197x, 1" 143, pl. I.XXXVIII; id., Els mosaics de paviment medievals a Catalunya, Barcelone, 1979, fig. 12 à 14 'n couleurs.

53 Informations archéologiques, dans Gallia, 33, 1975, p. 486-488, fig. 35, p. $4 \times 7$.

54 Cotamment le motif d'osselets de la gralerie nord-est, cf. supra, note 16.

55 Ci. supru, note 25.

56 Totamment dans les salles Cet E, le triclinium S du "Palais de Theodoric "à Ravenne, cf. F. Berti, Mosaici anlichi in Italia, Regione ollava, Ravenna I, n"s 33, 36 et 59 (pl. XXXI, XXXI, XLVII.

57 Belles vues en couleurs dans X. Barral, Els mosaics de paviment... ef. supra, note 52, fig. 10 et 11.

58 D.-J. Swiтн, Three fourlh-century schools of mosaic in roman Brilain, dans La mosaique gréco-romaine, I, Paris, 1965, p. 116, fig. 3.

59 A. Blaxco FreiJeiro, Mosaicos romanos de Merida, Madrid, 1978, p. 21 il 3.4, pl. 26. En Aquitaine, les exemples connus de rinceaux de cornes emboitees s'accompagnent d'un abondant feuillage ; je remercie .llle c.. Balmelle à qui je dois ce rensejgnement.

60 E. Barbier, La significalion du corlège représenté sur le couvercle du coffrel de Projecta, dans Cahiers Archéologiques abrégé : CArch, XII, 1962, p. 8 ; sur les compositions "héraldique's ", cf. F. Baratre, Le trésor d'argenterie gallo-romaine de Notre-Dame d'Allengon (.Maine-et-Loire), XLe Suppl. a Gallia, 19xl, p. 40, avec bibliographie. 


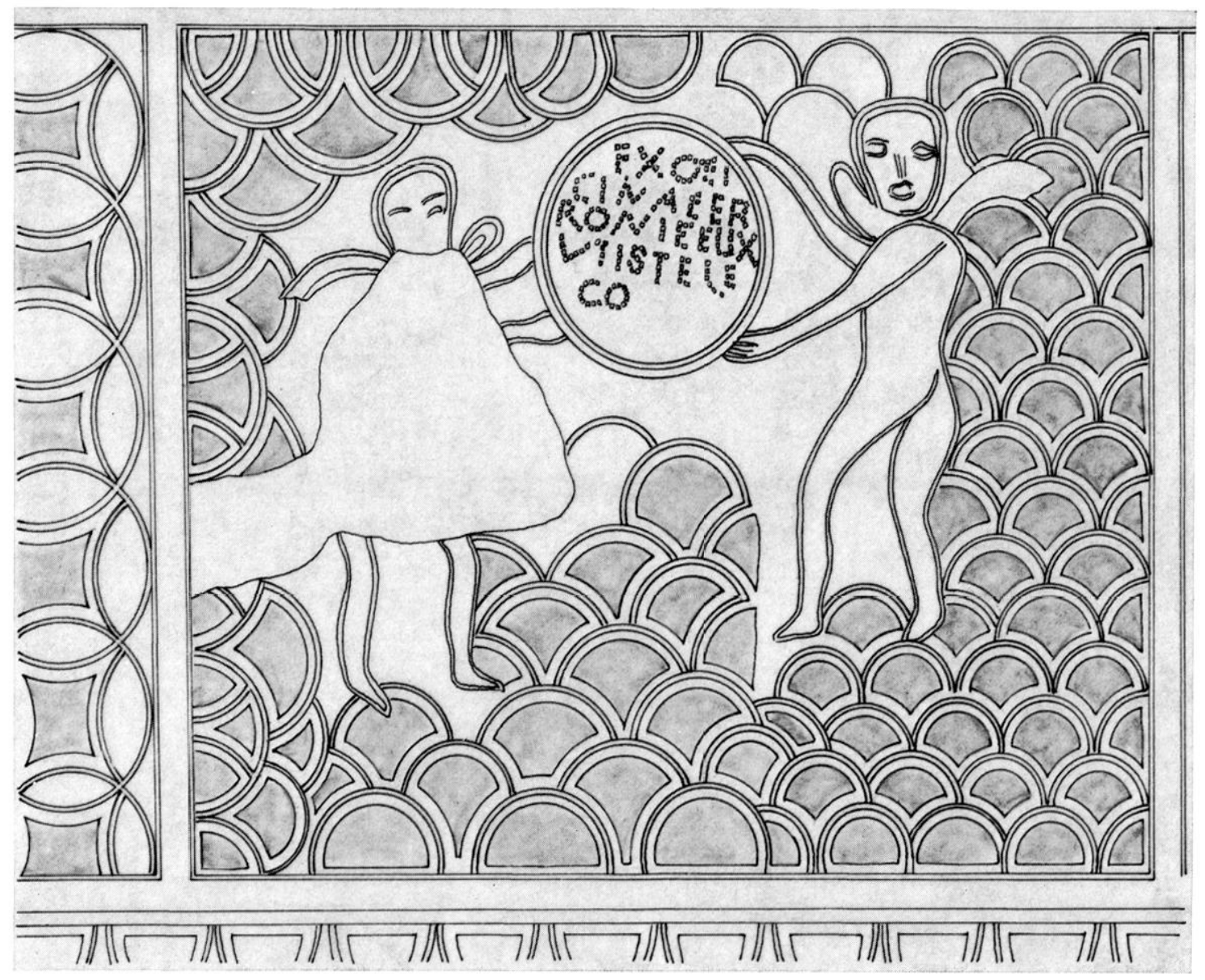

6 Dessin de Fillon (par R. Prudhomme d'après la photographie de M. Jusselinj.

Calendrier de $354^{61}$ où le cartouche du frontispice présenté par les pulti porte une acclamation au propriétaire, accompagnée de la signature de l'artiste. Cies représentations renvoient à des modèles très classiques dont la christianisation a assuré la survie jusqu'au $\mathrm{vi}^{\mathrm{e}}$ siècle au moins ${ }^{62}$; elles ne peuvent donc constituer un élément de datation.

Les mosaïques de l'aile méridionale.

Les deux autres mosaïques de la villa nous sont connues par des croquis at, pour la seconde, par quelques fragments dispersés dans trois musées.

61 II. STrk, Le Calendrier de 3;4, Paris, 1953, p. 120-123, pl. V' : Valentine lege feliciler. Voir aussi le pavement d'une annexe de la basilique chritienne d'Oued Remel Tunisie : P. (iacckler, Inventaire des mosaiques de la Giale et de l'Afrique, II, Paris, 1910, n" 463 'inscription perdue' et celui du triclinium $\mathrm{S}$ du "Palais de Théodoric "a Ravenne: F. BERTI, op. cil., no 60, pl. XLIX.

62 E. Kotzrivicr, Byzantine Art in the Maling, Londres, 1977, p. 39, émet l'hypothese de l'infiltration d'éléments de l'art imperial, comme l'imago clipeala, dans l'art chrétien, par le biais de la christianisation de l'Etal, des la fin du we siecle cf. fig. $75,154,176$. 


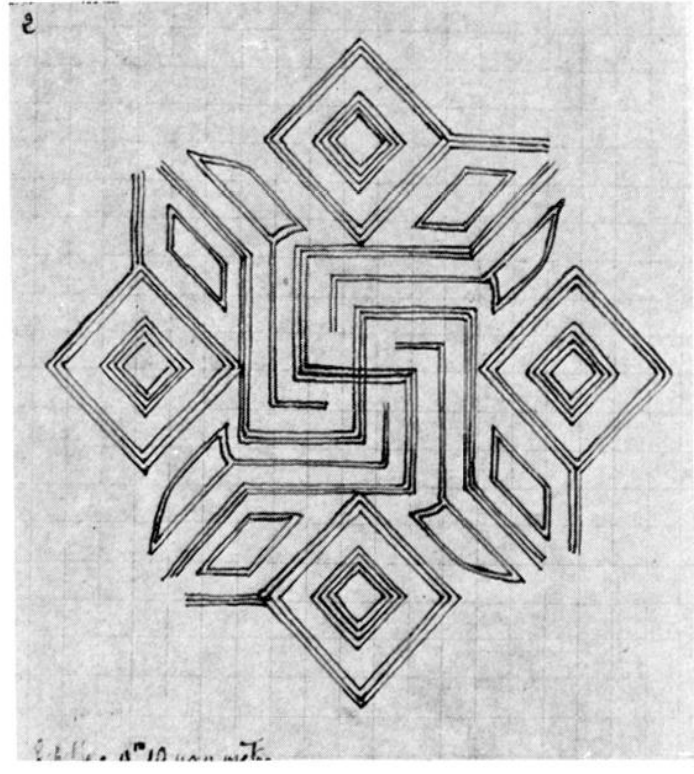

7 Mosaïque de la salle D dessin inédit de Lejeune reproduit par M. Jusselin).

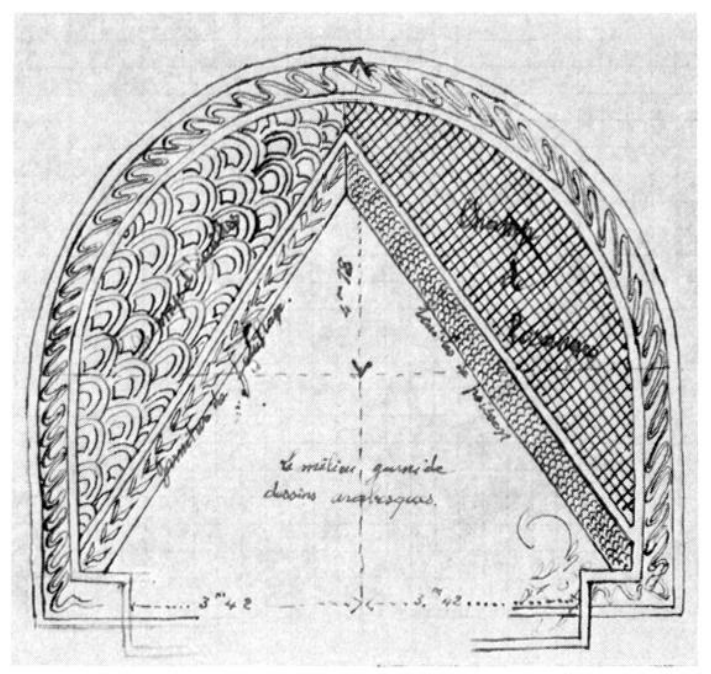

8 Mosaïque de l'abside de la salle C (dessin inédit de Lejeune).

La mosaïque de la salle $D$ ) (fig. 7 ) «figurait principalement de riches grecques d'encadrement et de champ, toutes d'une exécution assez soignée "63.

Le canevas constitue une variante, unique à ce jour, des étoiles de huit losanges tangentes par les sommets déterminant des grands carrés droits el des pelits carrés sur la pointe : les grands carrés sont traités en méandres de svastikas, les losanges partiellement ouverts ${ }^{64}$. Les motifs sont traités en filets simples, doubles ou triples, dont Lejeune a dessiné les joints entre les rangs de tesselles.

La mosaique de la salle $C$ (fig. 8, 9, 10). Le fragment le plus important se trouve au Musée de Chartres, ou le dessin reproduit par Vergnaud-Romagnesi m'a permis de l'irlentifier ${ }^{65}$.

Boisvillette semble n'aroir trouvé de vestiges de mosaïque que dans l'abside. "La mosaïque de lhémicycle formait un grand triangle décoré de volutes et de feuilles légères sur fond réticulé ${ }^{66}$." Le croquis de Lejeune ${ }^{67}$ (fig. 8) montre en effet un triangle isocèle inscrit dans l'abside, la base à la corde, le sommet touchant presque à la circonférence. Il

63 Bolsvillatre, Stalistique, p. 271.

6.4 Cropuis de lejeume, Ms. Jusselin, cahior $111, f^{\circ} \$ 39,9 \times 10 \mathrm{~cm}$; dimensions des motifs, deduites de l'echelle du dessin : grands carres, 33 à 35 cm, petits carrés, 19 a $20 \mathrm{~cm}$.

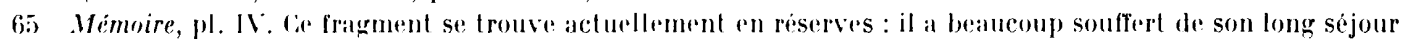
en plein air dans la conr du Musee. Il fut donné a la Societe archeologique d'Eure-et-Loir par le baron Reille, proprieLaje des terrains fouillés, cf. l'roces-rerbaux de la Sociélé..., I, 1861, séance du J4 octobre 1858, p. 121. Dans l'étal prósente par le dessin fin. l0it dapres une photographie prise en 1973 , il mesurait $0,71 \mathrm{ln} \times 0,60 \mathrm{~m}$.

66 Stalislique, p. 271 .

67 IIs. Jusselin, fo $87(12,5 \times 15) \mathrm{cm}$. 


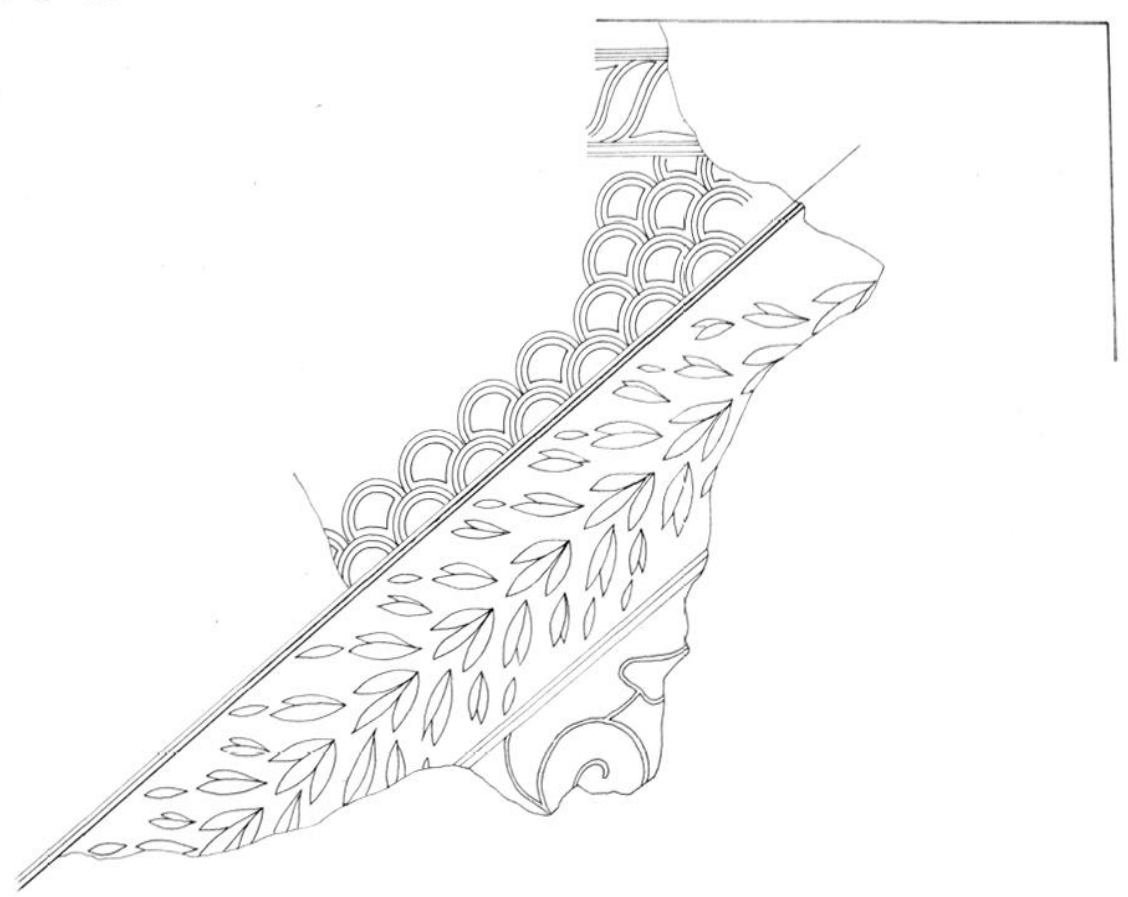

9 Fragment de la mosaïque de la salle c:, à l'angle de l'alsside : calque par R. Prudhomme de la planche IV b de Vergnaud-Romagnesi.

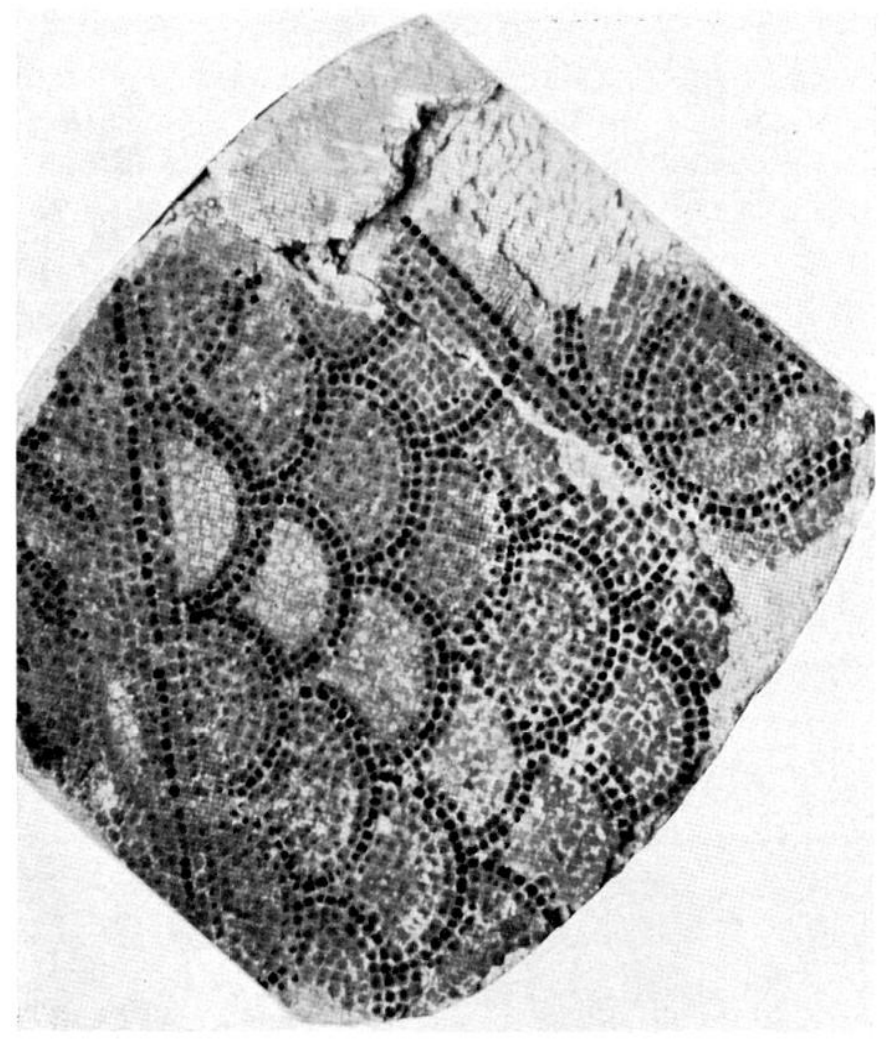

a

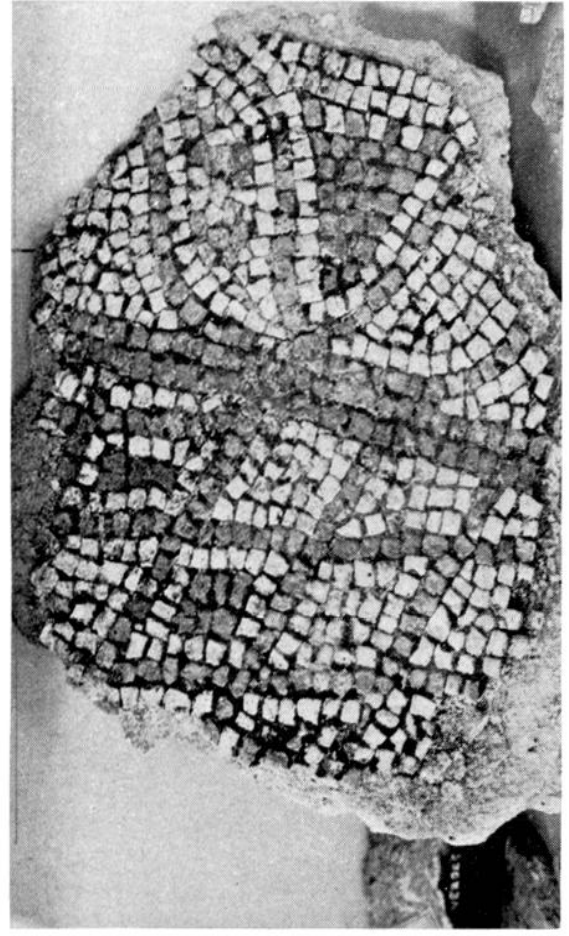

b

10 a, Fragment du .lusé de Chartres dapres photographie dr 1973; b, magment de chàtcautun. 
n'existe pas d'autre exemple, à ma connaissance, de découpage semblable d'une abside ${ }^{68}$. Mais cette disposition est la seule qui puisse expliquer l'angle de $38^{\circ}$ formé par la bande à ruban ondé et par celle à laurier dans le fragment de Chartres (fig. 10a), correctement relevé par Vergnaud-Romagnesi (fig. 9).

Le triangle central était garni de rinceaux à feuilles cordiformes, encore visibles sur le dessin de Vergnaud-Romagnesi. A gauche ${ }^{69}$, la portion de cercle porte des écailles au trait de $15 \mathrm{~cm}$ de diametre, emboîtant des écailles unies dont les couleurs se succèdent par rangées obliques, en rouge, beige jaunatre el gris bleu. Sur une bande large de $20 \mathrm{~cm}$, d'un beau rouge sombre, court une guirlande de laurier blanche aux feuilles si détachées les unes des autres qu'elles forment une sorte de jonchée orientée. L'autre portion de cercle paraît aroir été bordée d'une bande à petiles écailles. Le " champ de losanges » nous est conservé par les fragments d'Orléans et de Châteaudun (fig. 10 b) ${ }^{70}$. Chaque losange tracé en filets noirs $(9 \mathrm{~cm}$ de còté) en renferme un autre gris bleu, beige ou rouge. La bordure extérieure de l'absirle est conservée sur tous les fragments : c'est un ruban ondé aux faces alternativement gris bleu el gris beige, arec des calices tète-bèche rouges dans un sens, gris dans l'autre.

Boisvillette insiste sur la qualité de la technique, meilleure dans ce pavement que dans la mosaïque a inscription ${ }^{71}$. Les tesselles sont un peu plus régulières (de 8 a $12 \mathrm{~mm}$ de còté) ; toutefois la densilé ne dépasse pas 95) tesselles par $\mathrm{dm}^{2}$ dans le quadrillage losangé, $85 / \mathrm{dm}^{2}$ dans le ruban ondé, $75 / \mathrm{dm}^{2}$ dans la guirlande.

Comme dans la mosaïque de Steleco, les motifs sont tracés par des filets noirs, avec un filet blanc intérieur et un remplissage uni. Le ruban ondé est dépourvu de tout effet de relief. Très rare dans la Gaule centrale et septentrionale aux premiers siècles de l'Empire, ce décor linéaire a été l'un des motifs de prédilection des mosaïstes de l'Aquitaine à la fin de l'Antiquité ${ }^{72}$. Mais ce motif est connu de toutes les écoles de mosaïstes à la fin de l'Antiquité et serait un indice par trop ténu d'un apport des ateliers du Sud-Ouest, si l'hypothèse ne se trouvait étayée par la présence d'un marbre des Pyrénées gris pâle légèrement bleuté, marbre identique à celui du même ton utilisé dans les mosaïques de Taron et de Séviac--Yontréal ${ }^{73}$.

68 Les mosaistes de la fin de l'antiquite se contentent parfois d'adapter plus ou moins heureusement à la forme centree de l'abside des motifs isotropes à deux ou lrois axes, notamment dans la salle a sept absides de la maison de

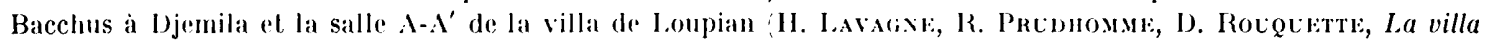
gallo-romaine des Pris-Bas à Loupian, dans Ciallia, 34, 1976, fiğ. 2 .

69) Il convient d'inverser le dessin de Verognaud-liomagnesi par rapport à celui de I.ejeune et au fragment de Chartres.

70 (Ont.jaxs : ${ }^{\circ}$ A 2251, don I. Jarry, 1849 - Bullelin de la Sociélé archéologique de l'Orléanais, I, 1819,

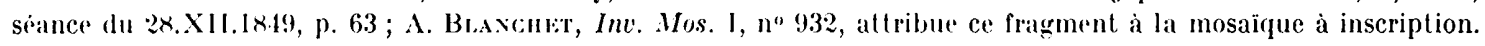

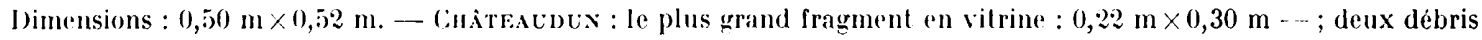
non jointifs inclus dans une plaque de ciment moderne, sous le preau d'entrie.

71 I.e supporl comporte trois couches : lit de pose blanc jaunatre à grain tres fin, $0,8 \mathrm{~cm}$ avec les tesselles, puis mortior de tuilean a grains fins $1-2 \mathrm{~mm}, 3 \mathrm{~cm}$, puis morlier plus grossier à gros grains de sable.

72 Voir la mise all point de C. Basmbirf, Recueil général des mosaïques de la Gaule, Xe Suppl. a Gallia, IV,

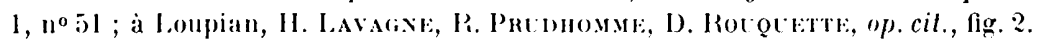

73 Examen en lames minces operé au Laboratoire de Pétrologie du centre de Recherches archéologiques du C....K.S. par II me II. Kieq; origine pyrénénne confirmée par II me G. Velde (Laboratoire de Pétrographie de l'Lniversite de Paris VI. 


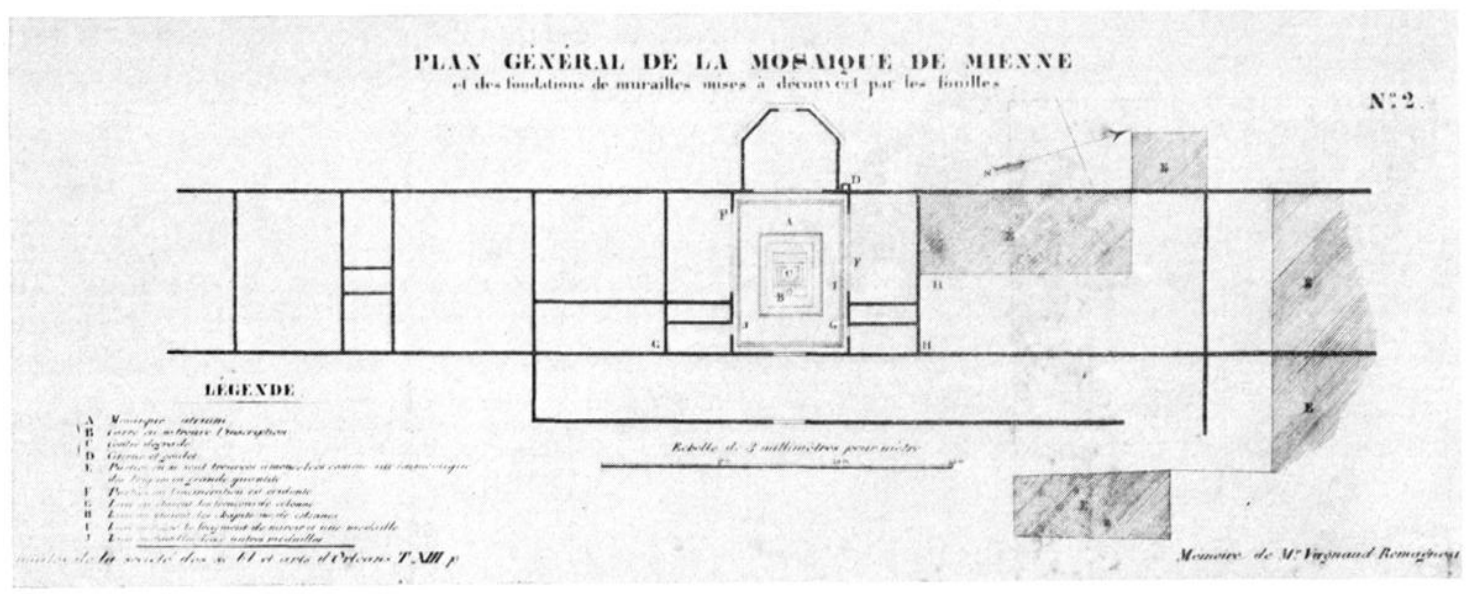

11 Plan de l'aile occidentale de la villa d'apres Vergnaud-Romagnesi, Mémoire.

L'architecture de la salle à la mosaïque de Sleleco (fig. 11).

Cette salle, d'amples proportions, ouv rait sur la galerie du péristyle, sur des dépendances latérale ${ }^{74}$ et sur une grande abside. Son sol de mosaïque se trouvait à $20 \mathrm{~cm}$ au-dessous des sols voisins; il était légèrement bombé, en pente vers un caniveau ménagé dans l'angle nord-est pour l'écoulement des eaux de lavage. Vergnaud-Romagnesi déduisait de ce dernier détail que le local était à ciel ouvert; mais Boisvillette remarquait que l'épaisseur d'un à deux mètres de débris déblaỵés au-dessus du parement et la présence dans ces déblais d'une grande masse de tuiles supposaient l'éroulement d'un toit. voire même d'un étage. Toutefois les murs, qui subsistaient sur une hauteur de $0,60 \mathrm{~m}$ i $1 \mathrm{~m}$, n’étaient épais que de $0,70 \mathrm{~m}$, ce qui exclut, notait-il, l'existene d'une voùte. Le support de la mosaïgue "reposait sur la terre végétale sans fondations", indique Boisvillette : l'hypothèse d'une superposition de pavements doit donc ètre écartée ${ }^{75}$. Le matériel recueilli est très rare : les débris d'un vase en marbre blane à godrons ${ }^{76}$, des "ferrements de portes et de croisées", un fragment de verre que Vergnaud-Romagnesi a pris pour un miroir, enfin trois monnaies, trois petits bronzes que Vergnaud-Romagnesi attribue a Gallien. Galerius llaximianus ot peut-être Victorin?7 recueilli au-dessus de la mosiïque, ce matériel ne peut contribuer à sa datation.

La forme de l'abside est plus caractéristique. Ouverte sur presque toute la largeur de la pièce, elle est en effet polygonale, à cinq pans dessinant une portion d'octogone. Les absides à pans coupés apparaissent dans les monuments rhrétiens de Ravenne dès la construction de la basilique d'l rsus à la fin du ve siecte et elles y restent en usage jusqu au

74 Locaux de service ot probablement longues couretles d'eclairage, sépares par un mur double; ces pieces étaient pavées de carreaux de terre cuite rectangulaires ot semi-circulaires : Borsvidertre, Notice. p. 156-157.

75 Ibid., p. 15j à 160; Boisvillelte note aussi la facture des murs, "en cailloux ordinaires, arec angles et baies revêtues en pierre crayeuse ".

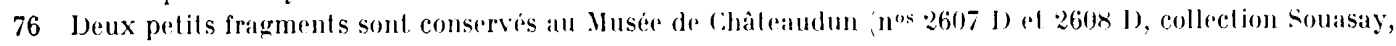
entrés le 4.VIII.1869.

77 Op.cil., p. 197 ; Bossvid.eTte, Nolice, p. 157, parle seulement de "denx petiles módailles en bronze, presque frustes, de 8 et 10 lignes ". 

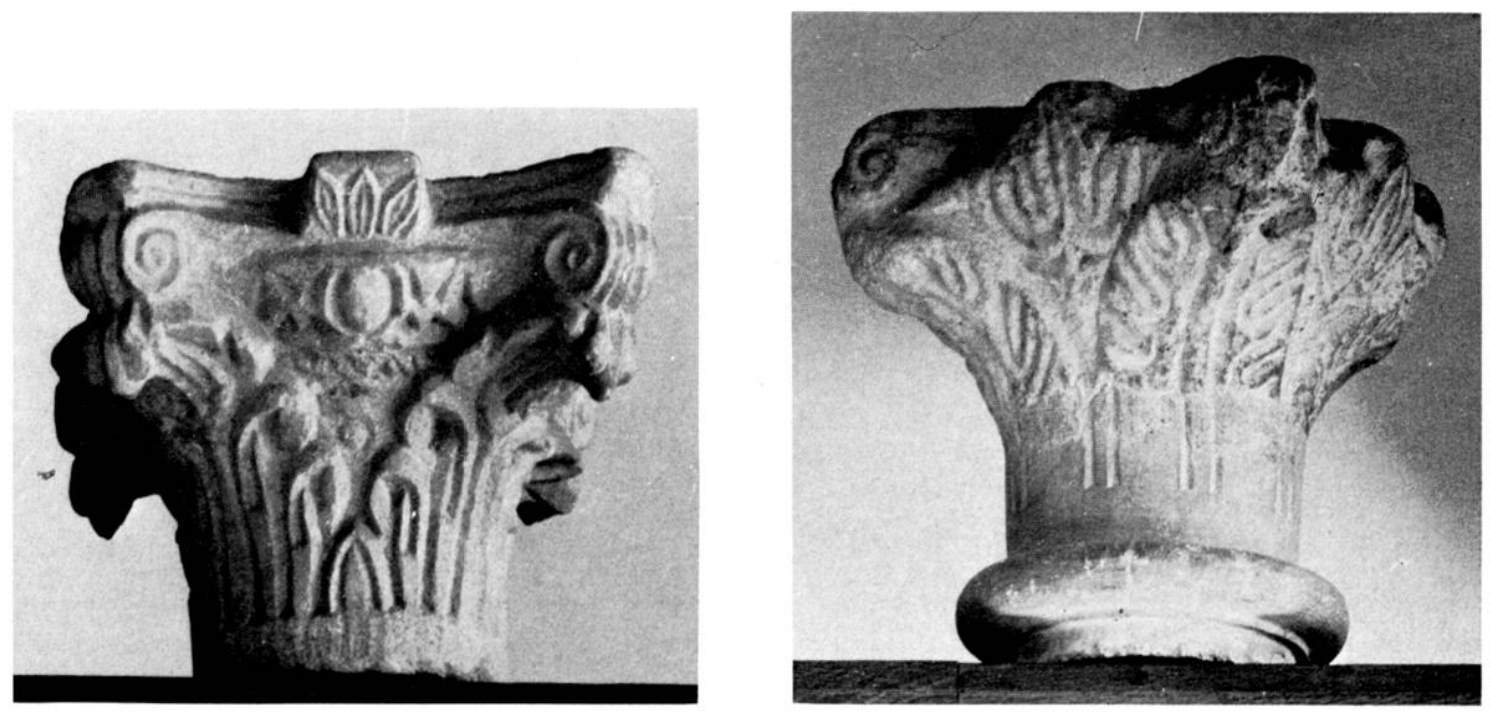

12 Chapiteaux provenant de la villa de Mienne, an Musée de Chateaudun.

$\mathrm{vI}^{\mathrm{e}}$ siècle; elles sont toutes, il est vrai, arrondies vers l'intérieur ${ }^{78}$. Nous trouvons des formes plus proches encore dans des villas d'Espagne occupées aux rve et ve siècles ${ }^{79}$ et en Novempopulanie, notamment à Lalonquette ${ }^{80}$, où un oecus a été reconstruit avec une abside demi-octogonale a la fin du rve ou au début du ve siècle, date assignée par les auteurs aux mosaïques.

Les comparaisons appelées par l'écriture et le style de la mosaïque de Steleco, ainsi que par la forme de l'abside de cette pièce, convergent vers le ve siècle et plus particulièrement vers la seconde moilié de ce siecle; il n'est pas exelu que ces deux éléments de décor ne soient venus enrichir, à cette période tardive, un oecus jadis plus simple ${ }^{81}$.

Les chapileaux (fig. 12).

In dernier élément de décor appartient lui aussi à une période tardive de l'existence de la villa. Il s'agit des fragments architecturaux découverts sur la mosaïque ou dans ses abords immédiats : en $($ (fig. 11) des fragments de fûts en marbre "ordinaire " ou "gris veiné de blane ${ }^{82}$, deux bases, enfin deux chapiteaux au nord de l'oecus, en $I I$ (fig. 11).

Comme pour l'inscription, le problème chronologique a étè méconnu. VergnaudRomagnesi et Boisvillette n'ont fait que mentionner l'existence des chapiteaux.

is (i. Bovis, Storia e architellura degli edifici paleocristiani di cullo di Ravenna, Bologne, 1964, 111, 4, p. 116

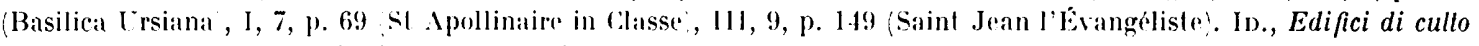
d'èta palencristiana nel lerritorio Ratennate di Classe, Bologne, 1969, p. 1, 6, 51, 56, 69, 195, 213.

79 J.-G. Ciontiws, Les willas hispano-romaines, Paris, 1979, pl. V, p. 355 (Aguilafuente, prov. de Ségovie), pl. LIII, p. 437-438 Adaja, prov ar Valladolid, pl. XXX, p. 35l (Sabada, abside ajoute ot mosaiquée).

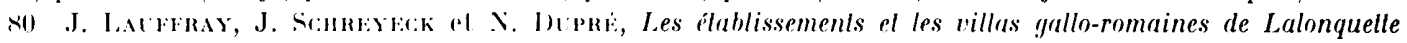
(Pyrénées-Allantiques), dans Ciallia, 31, 1973, 1, p. 146-1.47, plin tig. 20, p. 1.4.

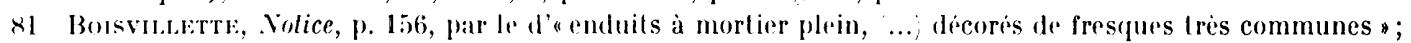

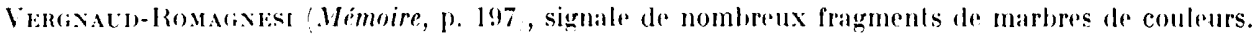

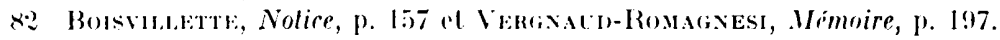


A. de Caumont, qui les a publiés en $1856^{83}$, a certes bien reconnu leur style "mérovingien "; cependant, pour justifier cette anomalie, il les a attribués non pas à la villa, toute romaine à ses yeux, mais au petit fanum découvert à l'ouest de Narbouéé, qu'il a interprété comme un édifice paléochrétien.

Ces fragments architecturaux sont conservés au Musée de Châteaudun ${ }^{85}$. Ils proviennent de supports de faible diamètre -- $0,20 \mathrm{~m}$ à $0,23 \mathrm{~m}$ pour les fùts ${ }^{86}$, dimensions comparables pour les chapiteaux -..., trop grêles pour avoir pu porter des linteaux. On supposera donc qu'ils appartenaient soit à un édicule ${ }^{87}$, soit à une arcature décorative, dont il est difficile de déterminer l'emplacement précis, en raison des bouleversements subis par les ruines avant leur sauvetage par Boisvillette. L'un des chapiteaux conservés, incomplet, est en marbre blanc grisâtre à grain assez grossicr; l'autre est taillé dans un marbre blanc plus fin, permettant une facture plus fouillé $e^{88}$. Tous deux, avec leurs quatre feuilles d'angles au-dessus desquelles saillent des volutes assez écrasées, appartiennent à un groupe représenté au Baptistère de Poitiers et à Béziers ${ }^{89}$. Ils s'apparentent également à un petit chapiteau de marbre de Saint-Denis, dont il n'est pas certain qu'il provienne de la basilique élevée par sainte Geneviève à la fin du ve siècle ${ }^{90}$. En Aquitaine, "des ateliers de sculpteurs de chapiteaux de marbre ont fonctionné sans interruption depuis la fin de l'époque romaine", écrit J. Hubert" ${ }^{91}$. Toutefois le style des chapiteaux apparentés à ceux de Mienne est considéré par les spécialistes comme "très évolué ", ce qui exclurait toute datation antéricure au vir ${ }^{\mathrm{e}}$ siècle ${ }^{92}$.

Saint Avenlin el la villa de Mienne.

C'est cependant sur un argument littéraire que se fondait Boisvillette pour supposer la survie de la villa au début du ve siècle; c'est, paradoxalement, l'argument le plus incertain. Il est apporté par un passage de la Vie de sainl Aventin connu des érudits du Xıx siècle par l'intermédiaire de l'Ilisloire sommaire du Dunois, de l'abbé Bordas ${ }^{93}$.

Entre 485 et 490 , Aventin se rend de Chartres à Chateaudun pour prendre possession de son siège épiscopal. L’abbé Bordas écrit : "Notre légendaire suppose qu'il fit la roule à pied, car il le fait

83 Dans Bullelin Monumental, XXII, 1856, p. 475-47x dessins communiqués par Boisvillelle, p. 475 ot 476.

84 Altribution reprise par D. Fossaro, Iees chapiternx de marbre du VII siécle, slyle el ćvolution, dans CArch, III, 1947, p. 70 , note 3 .

85 Auquel Boisvillette les a donnés en 1865 ; cf. A. Noltil, op. cil., p. 10.

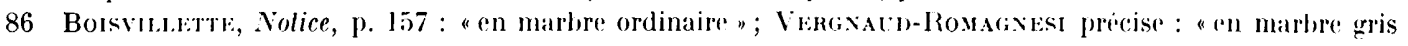
veiné blanc "(Mémoire, p. 197).

87 Cf. Bultetin de la Societé nalionale des Anliquaires de France, séance du 2 mai 1979, hypolhes: de ... Juval selon laquelle ces supports pouraient appartenir à un ciborium, an-dessus d'un aulel place au centre de la mosaïque de Steleco, et attesteraient la transformation de la piece en un sanctuaire chrétien. Le hiatus d'au moins un siecte: entre les datations possibles pour les mosaïques et pour les chapiteaux oblige à ecarler cette interessante hypothèse.

88 l)imensions : 1) diam. : 0,154 m (à la cassure, larg. à l'abaque : $0,225 \mathrm{~m}$ el $0,2 \times 5 \mathrm{~m}: ; 2$ diam. :0,174 m, larg. à l'abaque : $0,330 \mathrm{~m}$, haut. $0,240 \mathrm{~m}$.

89 D. Fossari, op. cil., p. 71, 7., 80 note $6 \nmid, 82$; fig. 10, p. $\times 1$.

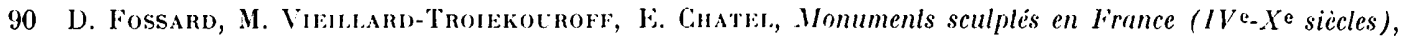
I, Paris el son département, Paris, 1978, no 314, p. 159-160, pl. XCi.

91 Ibid., Préface, p. xiv-xv.

92 Ibid., p. 157-158.

93 Rédigé à la fin du xvme siècle, restie manuscrile; editée a chartres en $1 \times \times 1$. 
se reposer à cause de sa lassiluile à Mlianne, hameau dont il reste quelques maisons près Marboy ". L'auteur indique sa source: "Le plus ancien détail qui nous soit resté de ce Saint Évêque ne date cependant que du treizieme siècle, à ce que l'on présume. C'est une légende en vieux vers français composés par un ecclésiastique de Chàteaudun dont on ignore le nom ". Ce manuscrit, transmis aux Bollandistes au xrmu siècle, a disparu ${ }^{94}$; les . Icta Sanctorum ne donnent de la Vie de Saint Aventin qu'une version abrégée. qui ne nomme pas le lieu où saint Avent in s'arrète el bénéficie d'une vision ${ }^{95}$. L'abbé Bordas, qui ne connaissail de Mienne que le hameau moderne élabli d'ailleurs sur les fondalions de la villa, cf. ligr. 11 .., ne pourait ètre tenté de faire coïncider sa source avec une réalité archéologique encore insoupçonnée.

L'examen de la carte du site de Mienne et Marboué, dressée en 1933) par V. Jusselinn, me paraît démontrer que la halte d'Aventin à Vlienne ne se justifiait que par la survivance de la villa dans les dernières années du ve siècle et confirmer, en retour, la véracité de la tradition.

\section{Le sile de Mienne-Marboué (fig. 1:3).}

La voie romaine de Cihartres a Tours par Châteaudun, dont le tracé est bien reconnu de Bonneval à Marboué, passait au sud-est de Marboué et franchissait le Loir au niveau du vieux pont détruit en 1834. Au nord-ouest de la voie s'étendaient, sur $440 \mathrm{~m}$ de longueur ${ }^{97}$, les immenses bâtiments de la villa rustica, partiellement dégagés par Boisvillette, organisés autour d'une seconde cour indépendante du péristyle de la villa urbana, précédés par le fanum dont le plan a été publié par Vergnaud-Romagnesi ${ }^{98}$. Pour se rendre à Mienne, Aventin devait quitter la route et longer le Loir vers l'ouest sur $500 \mathrm{~m}$ : on voit mal ce qui eùt justifié un tel détour, sinon l'existence de la villa.

Le nom même de Yarboué transmet l'information de l'existence d'un grand domaine, possédé à une époque indéterminée, par un propriétaire portant le nom germanique de Marbod ${ }^{99}$. Au sud-est de la voie romaine, dans le Champ des Cercueils, non loin du fanum et d'une chapelle Saint-Martin détruite au $\mathrm{xvI}^{\mathrm{e}}$ siècle, on a découvert en 1862 (lors de la construction du chemin de fer) une nécropole mérovingienne ${ }^{100}$; en l'absence d'une fouille

9.1 Ibid., p. 65, p. 62-63. Bordas Ini-même no comnaissail ce poime que par une traduction latine antèrieure a 1601, elle-même disparue depuis lors; le R. P. Van der Stracten a bien voulu me confirmer qu'aucune trace du manuscrit ne subsiste dans les fichiers de la Societé des Bollandistes.

95 De $S$. Aventino Episcopo Castrodunensi in Gallia... ex rythmo gallico succinle concinnata, 4 fevvier, I, \$5, p. 485. Sur le siège épiscopal d'Aventin, cf. L. Ducuesse, Fasles épiscopaux de l'ancienne Caule, II, Paris, 1910, p. 425.

96 Cahier III, fo 93 ; repris par H. I.I\%, op. cil., 1) 108.

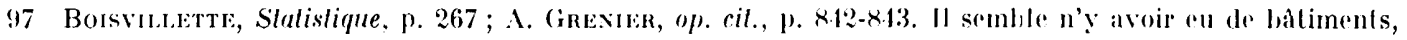
profonds de $10 \mathrm{~m}$, que sur le cote nord de l'enclos, dont la laregenr alleignait lof m et la superficie environ 6 ha. les dimensions baglent la villa de Mieme-.Marboue aux plus grands elablissements decouverts diuns le Nord et le lentre de la France : cf. R. Gowtry, Recherches aíriennes de la Loire an Rhin en 1976, dans Imossiers de l'Archéologie,

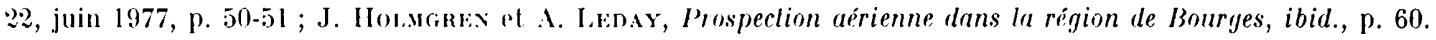

98 Mémoire, pl. IV, p. 293-296; Borsvul.fetrs, Nolice, p. 16.1-166, stalistique, pl. 272.

99 Abbé J. Vita.eTre, Marboú, Dérivé gallo-romain descriptif ou dérivé d'un anthroponyme?, article ronéotypé dépose aux Archives nationales, 1967, fos $\times$ e $17-19$.

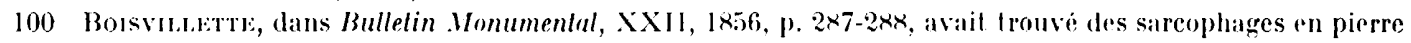
lendre du pays el de rares objels. Cf. A. nF Brifort, Noles sur les Anliquilés décomerles à Marboú, dans Bulletin

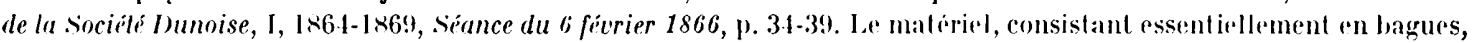
en boucles et plaques de ceinturons in bronze ot argent) et en quelques armes " couleaux ", fer de lance, n'est que tres partiellement conservé au Ifusé de Chàleaudum; le contenu de la lombe la plus richre, ourerte en 1865 , avec une

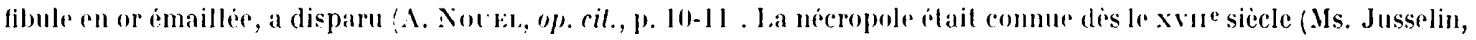
fo 117 , qui cile un registre paroissial de 1611 . 
MICHELLE BLANGHARD-LEMÉE

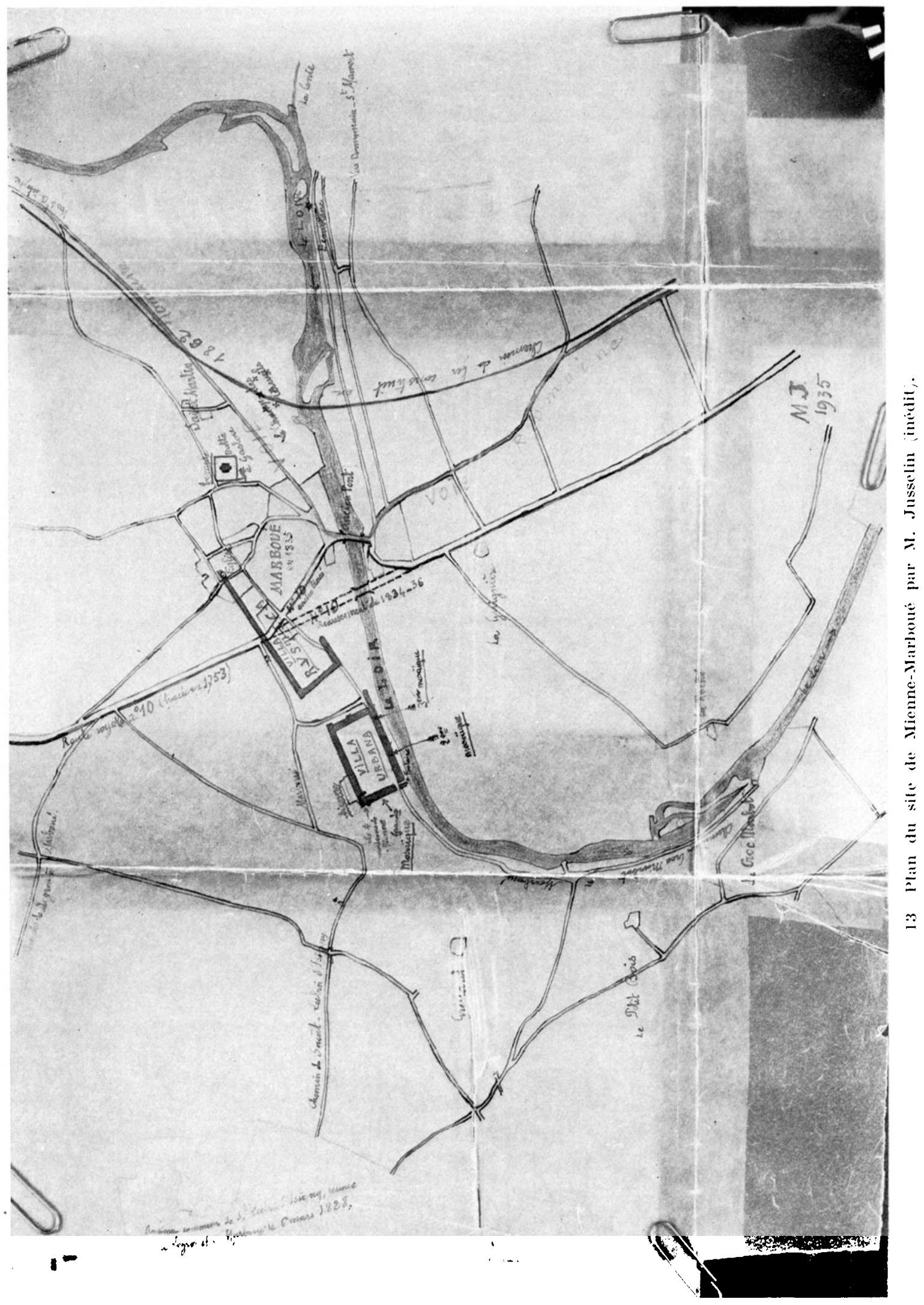


méthodique, il est impossible d'en déterminer avec précision la chronologie. Mais on reconnaitra ici le phénomène bien décrit dans les plaines de Picardie par R. Agache, qui l'attribue à la période des invasions germaniques : le déplacement de l'habitat par la création de villages auprès de la route, et non à l'écart comme le sont les villas romaines ${ }^{101}$. On voit à Mienne-Marboué que ce déplacement n'implique pas la disparition de la villa; il pourrait s'expliquer par l'implantation d'un établissement de lètes entre la route et l'enclos de la villa rustica ou à Saint-Martin. Ce mode de peuplement est assez répandu dans toute la région entre Le Mans et la vallée de la Loire, où il a été étudié par H. W. Böhme102. Le chef de cette colonie militaire barbare aurait pu prendre possession de la villa dans le courant du ve siècle ou après la défaite de Syagrius en 486.

L'étude des documents que nous possédons encore sur la villa de Mienne-Marboué fait apparaître un certain nombre de preuves de la survie de cette importante villa après les invasions du début du ve siècle et de son occupation par un propriétaire germanique qui en a fait remanier le décor. Dans l'état actuel des recherches sur les divers aspects du décor architectural, nous sommes obligée d'admettre deux phases dans ces travaux d'embellissement, la première correspondant aux mosaïques et à l'abside polygonale qu'il est difficile de dater plus tard que la fin du ve siècle, la seconde aux chapiteaux, que leur style ferait attribuer plutôt au viI ${ }^{\mathrm{e}}$ siècle. Des découvertes ou des travaux récents au nord de la Loire ${ }^{103}$ ou au centre de la France ${ }^{104}$ permettent maintenant d'affirmer que ce cas n'est pas isolé. Les données archéologiques, si incomplètes soient-elles, viennent ainsi éclairer d'un jour nouveau de larges pans de l'histoire de la Basse-Antiquité laissés dans l'ombre par les textes anciens. Les mosaïques témoignent, pour leur part, de la persistance d'une activité artistique dans le sud-ouest du Bassin Parisien après le début du ve siècle et de la continuité de relations culturelles avec l'Aquitaine.

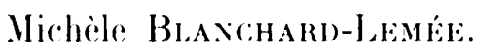

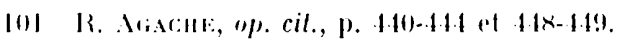

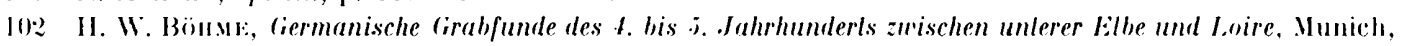

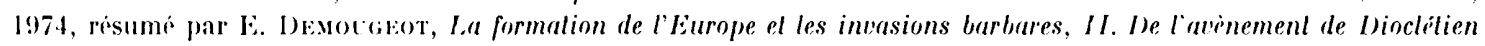
an début du l'le siecle, fasc. 1, Paris, 1979, p. 273-274.

103 Deux villas du Val de I.oire comportent des pavements au moins aussi lardifs que celui de Vlienne: celles de Châtigny-fondeltes et de Saint-kemy-la-Varenne article en préparation ; voir aussi la mosaïque de Nigennes,

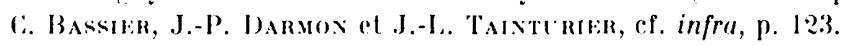

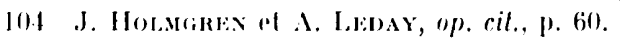

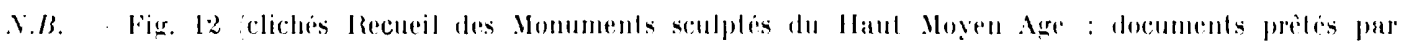

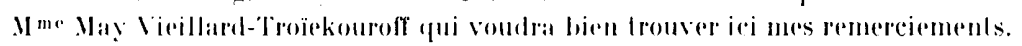

